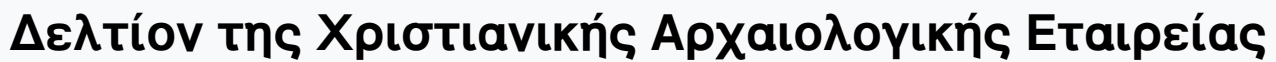

Tó 14 (1989)

$\Delta \varepsilon \lambda$ tíov XAE 14 (1987-1988), Пврі́обос $\Delta^{\prime}$

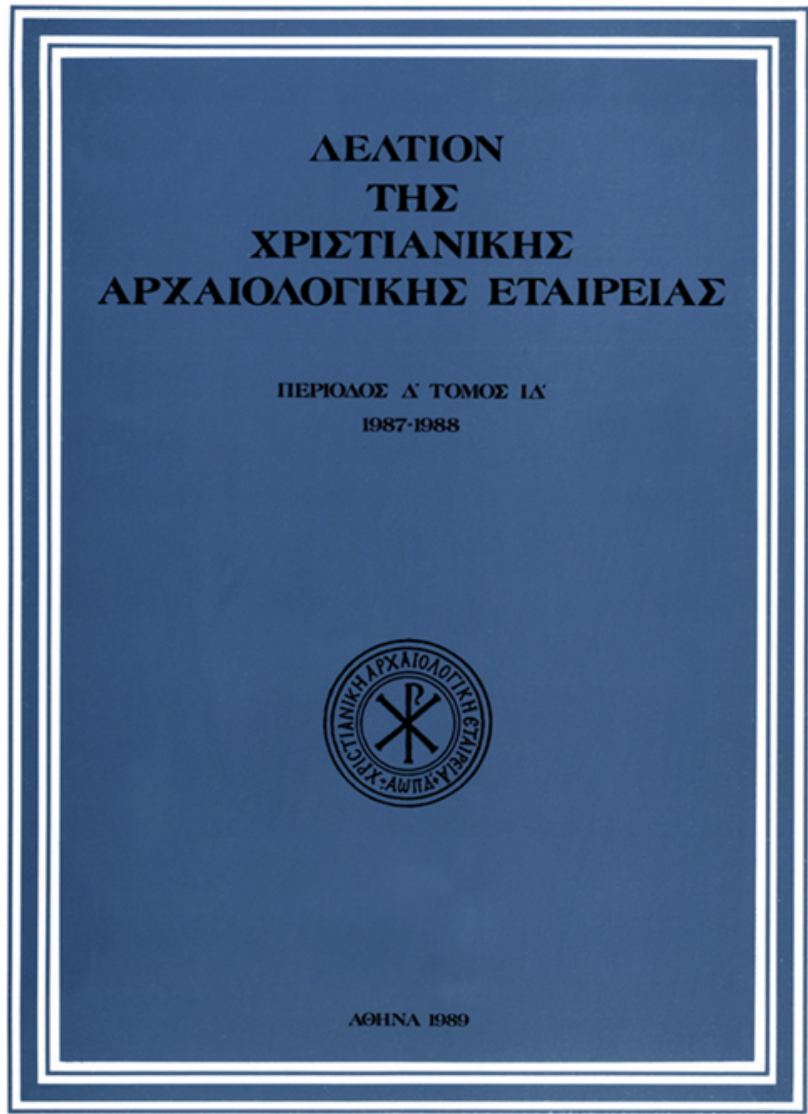

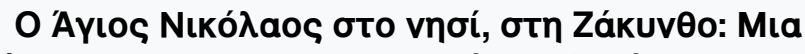

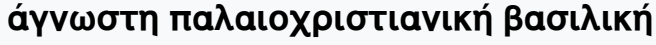

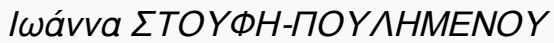

doi: $10.12681 /$ dchae.1016

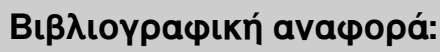

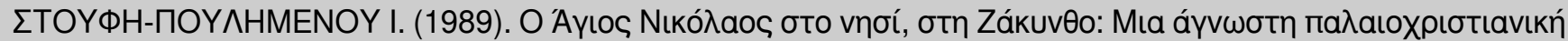

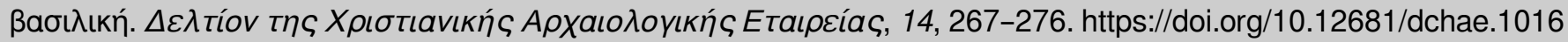




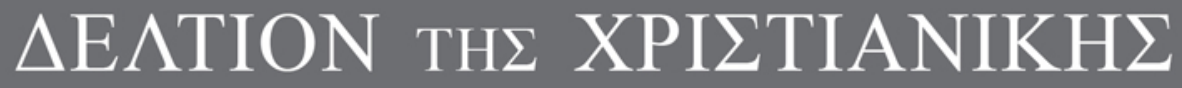 APXAIO $\Lambda$ OГIKH $\Sigma$ ETAIPEIA $\Sigma$}

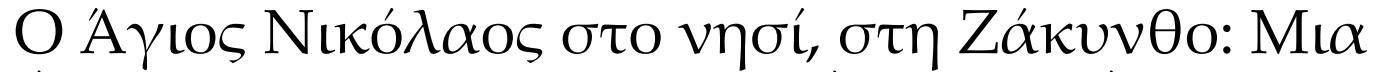

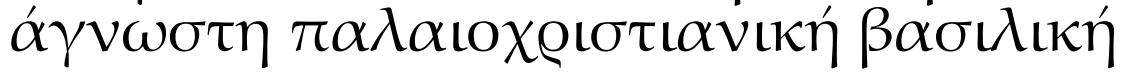

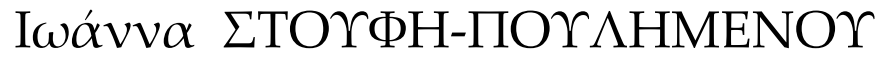

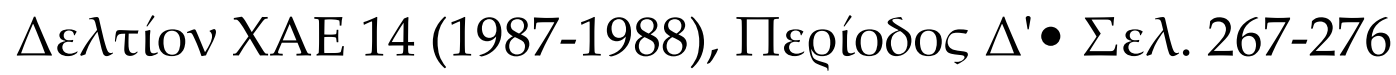

A@HNA 1989 


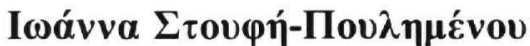

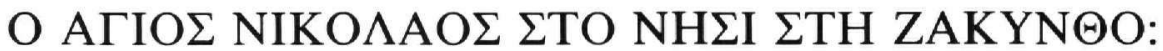

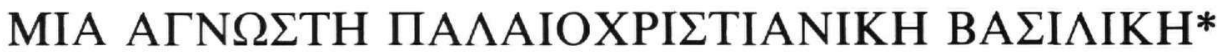

$\sum$

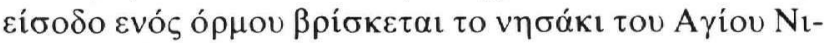

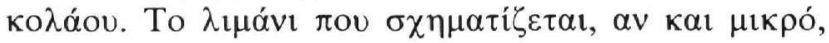

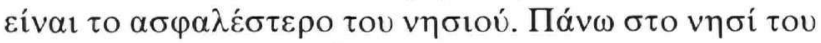

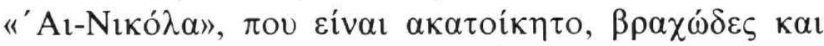

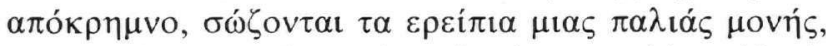

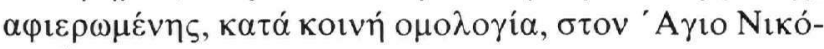
$\lambda \alpha \mathrm{o}^{2}$.

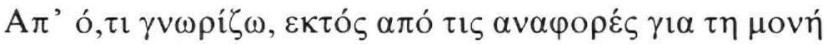

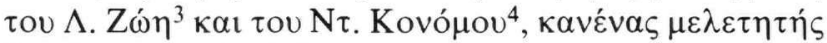

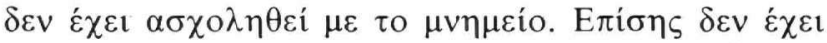

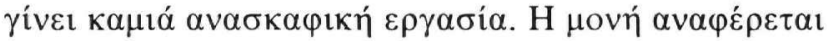

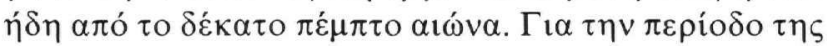

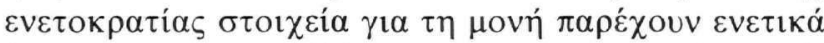

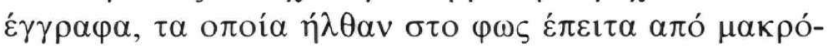

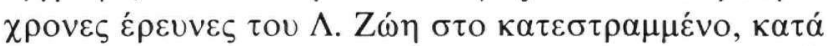

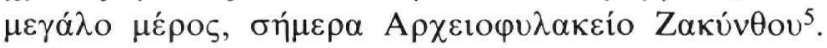

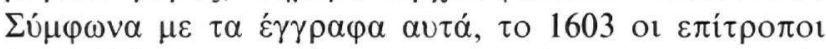

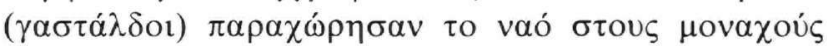

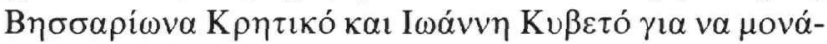

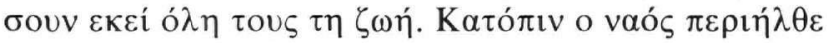

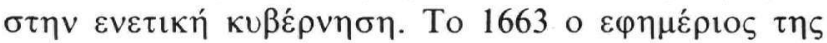

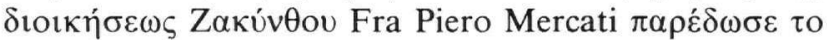

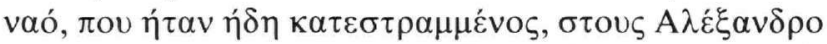

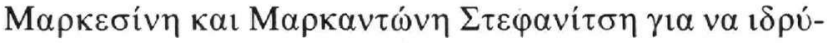

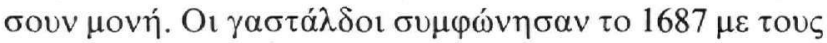

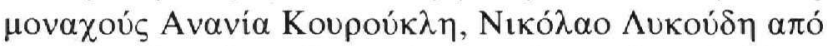

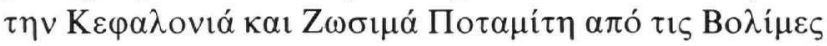

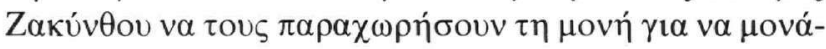

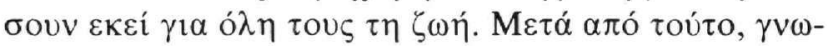

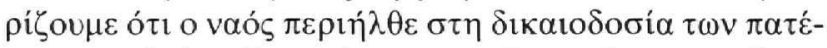

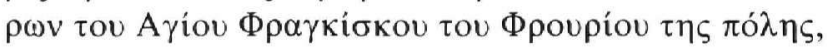

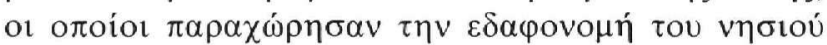

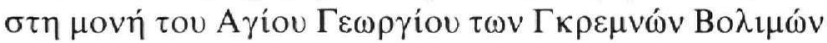

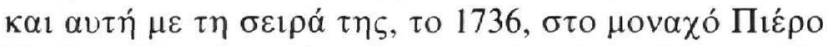

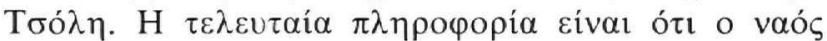

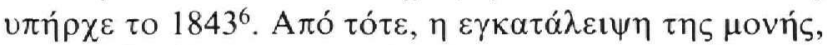

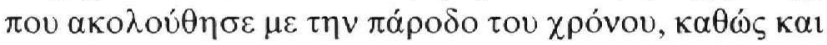

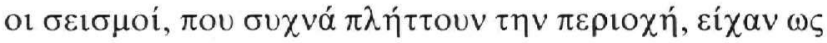

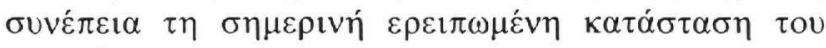
$\mu \nu \eta \mu \varepsilon i ́ o v$.

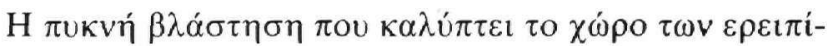

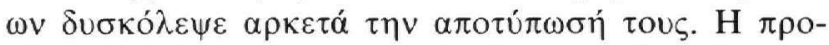

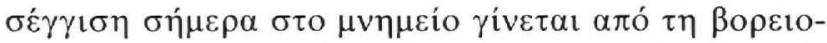

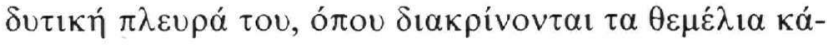

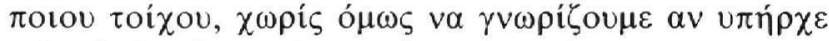

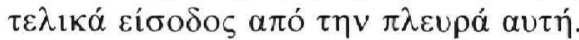

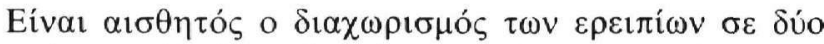

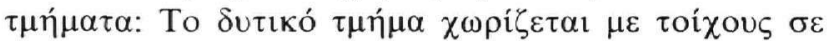

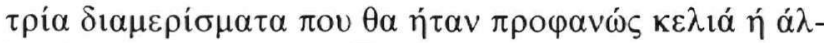

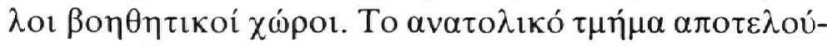

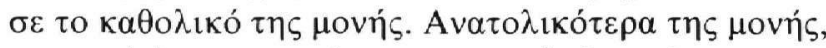

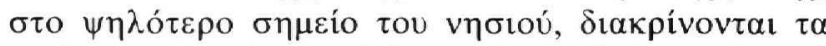

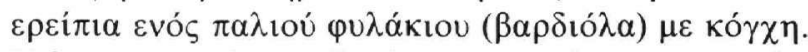

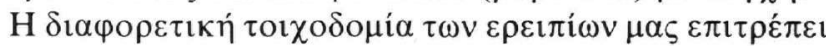

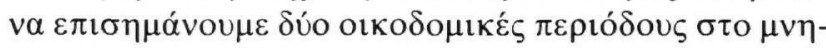

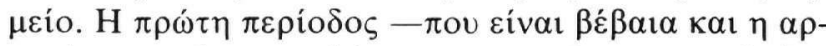

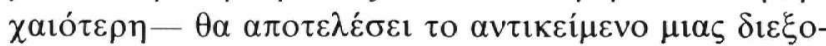

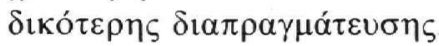

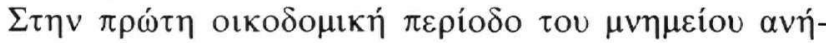

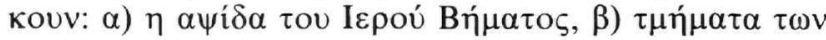

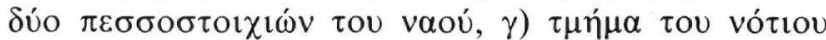

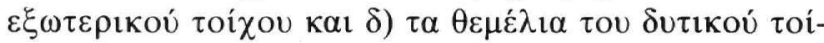

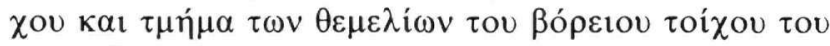
vaoú (Eıк. 1).

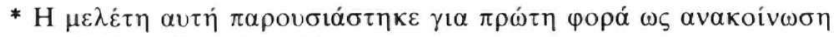

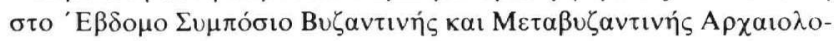

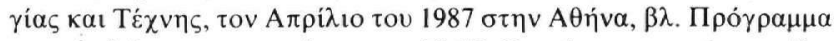

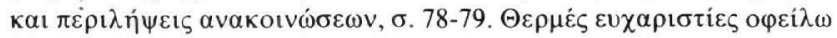

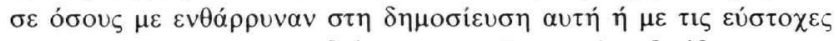

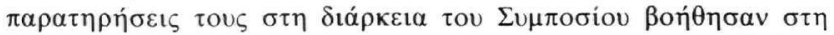

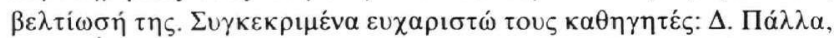

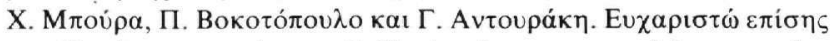

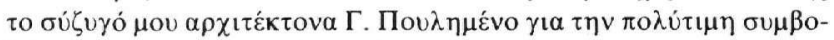

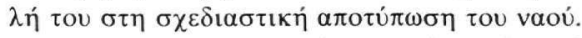

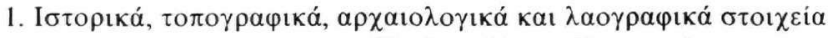

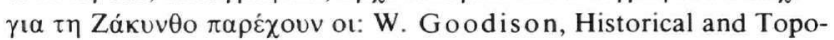
graphical Essay upon the Islands of Corfu, Leucadia, Cephalonia, Ithaca and Zante, London 1822. O. Rie m a n n, Recherches archéologiques sur les Iles Ioniennes, III: Zante, Paris 1879-89. Erz Salvator,

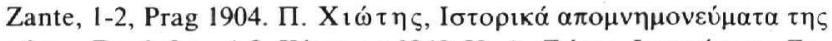

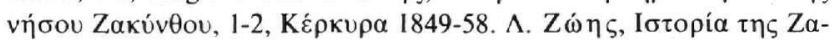

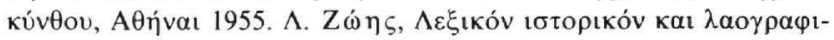

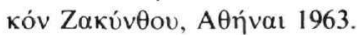

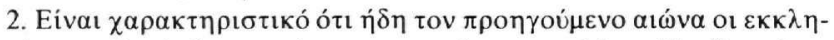

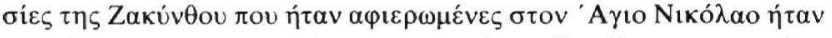

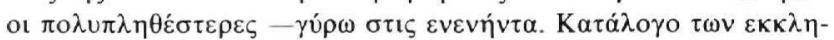

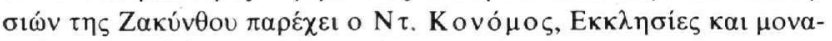

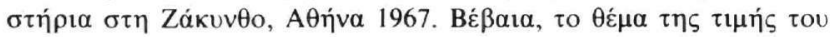

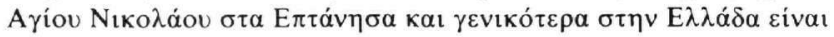

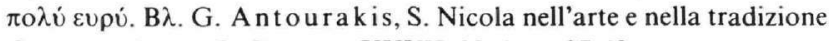
Grecoortodossa, OrCr, anno XXVII, N. 1, б. 37-43.

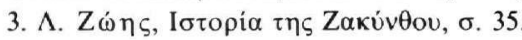

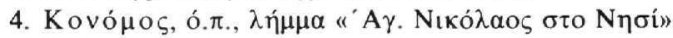

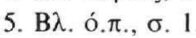

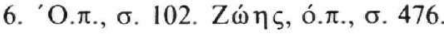



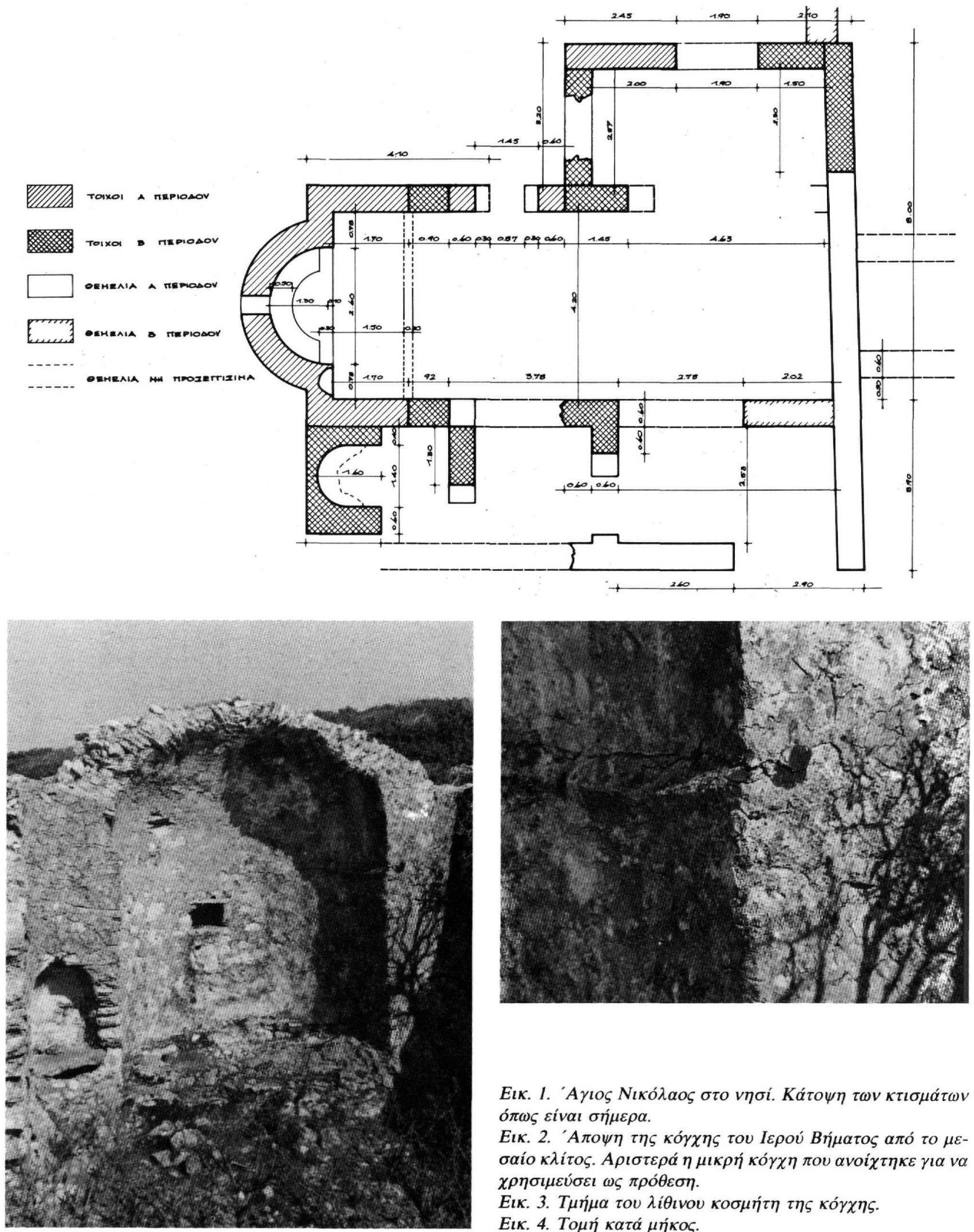

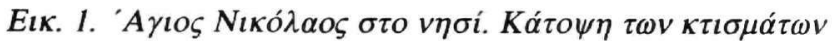
ó $\pi \omega \varsigma$ sival $\sigma \dot{\eta} \mu \varepsilon \rho \alpha$.

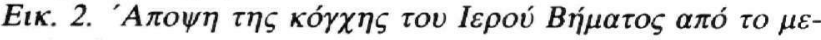

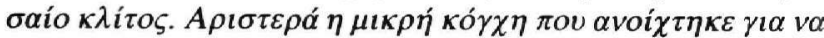

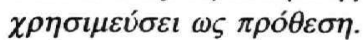

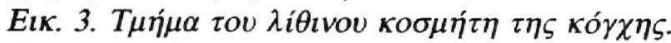

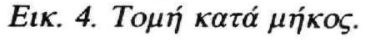




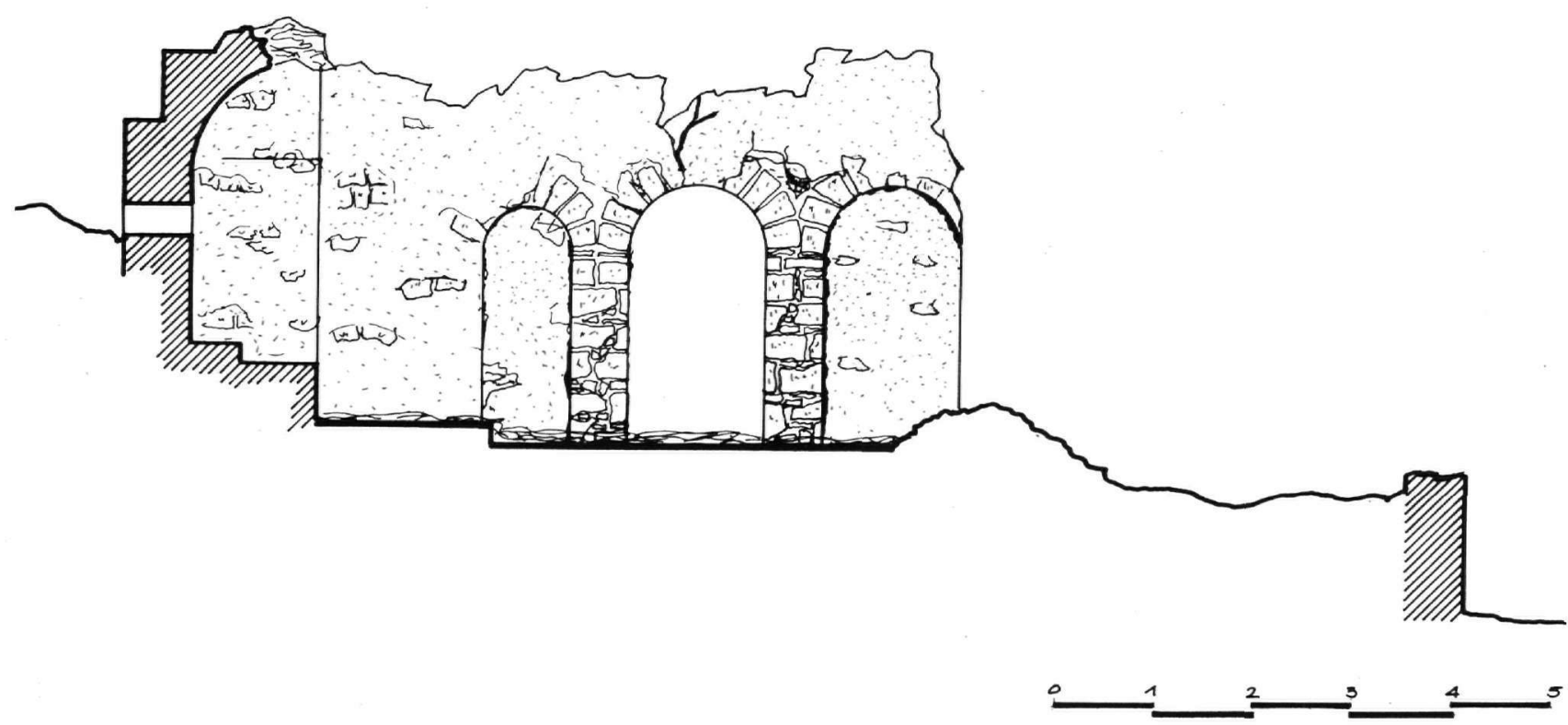

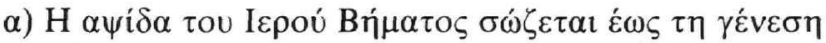

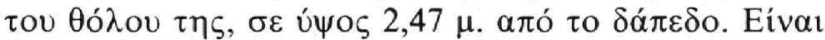

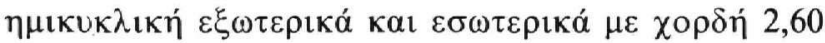

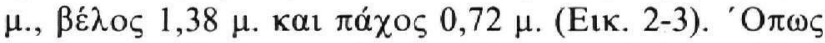

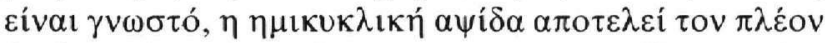

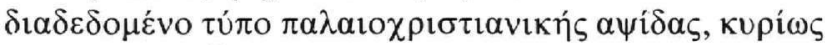

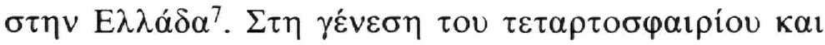

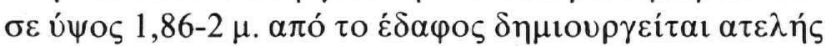

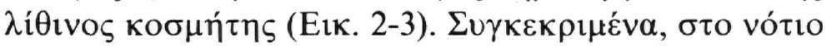

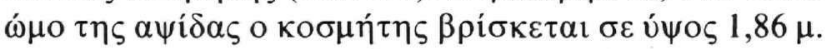

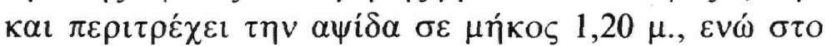

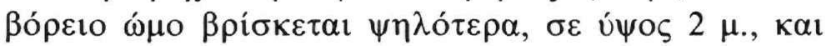

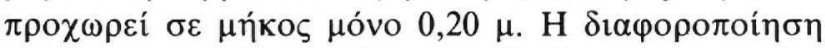

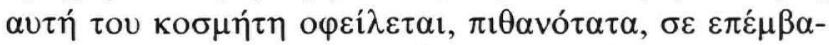

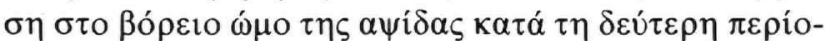

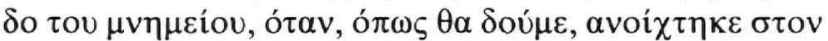

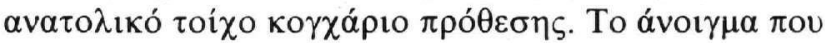

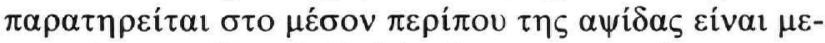

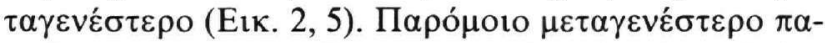

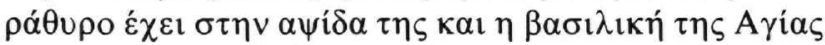

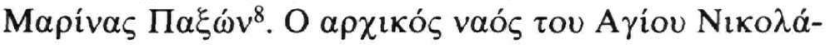

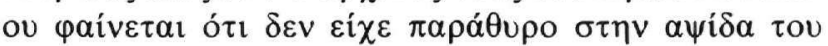

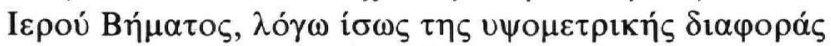

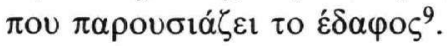

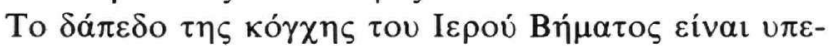

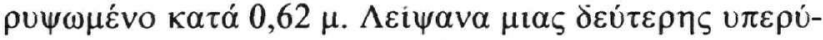

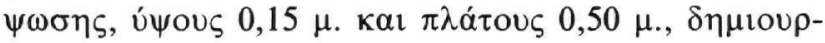

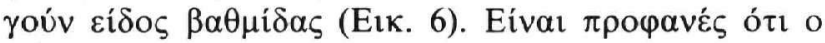

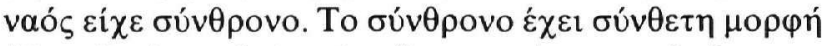

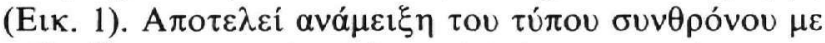

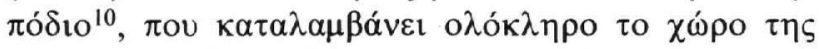

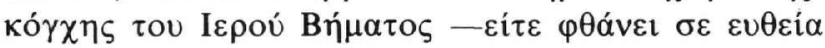

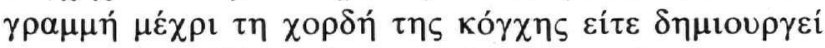

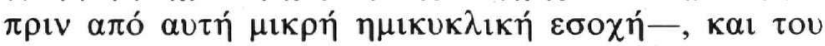

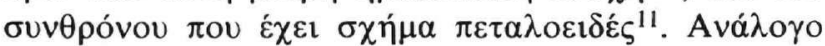

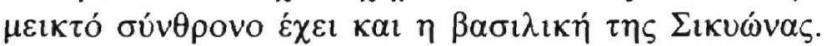

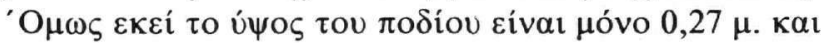

7. B $\lambda$. Ch. Delvoye, Etudes d'architecture paléochrétienne, II. L'ab-

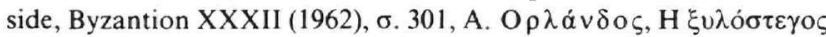

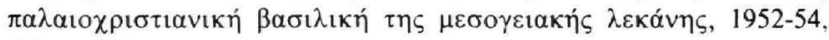
$\mathrm{A}^{\prime}$, , .208

8. П. Вокото́ $280 \alpha-\beta$.

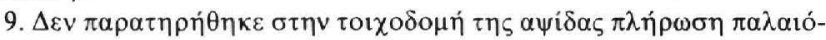

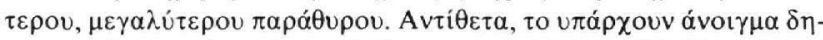

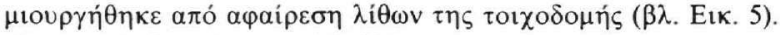

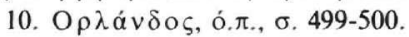

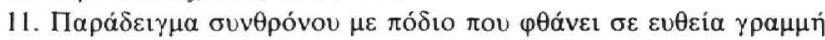

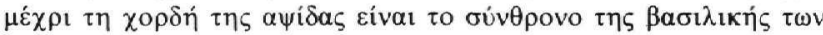

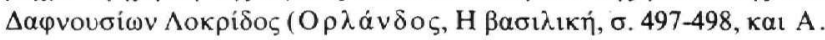
Orlandos, Une basilique paléochrétienne en Locride, Byzantion V

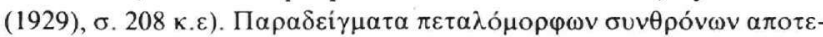




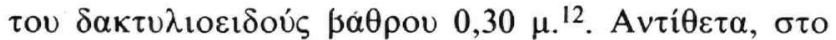

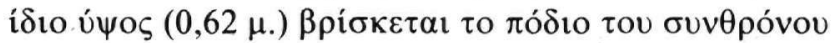

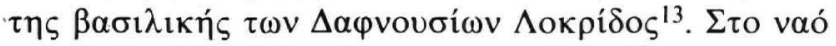

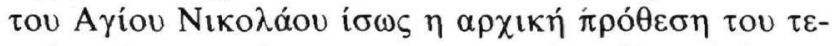

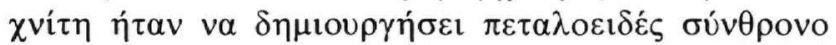

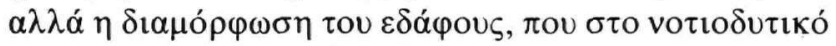

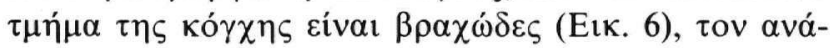

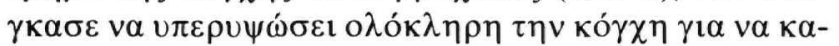
$\lambda u ́ \psi \varepsilon \imath$ to $\beta \rho \alpha ́ \chi o$. 'O

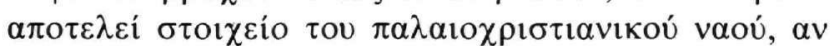

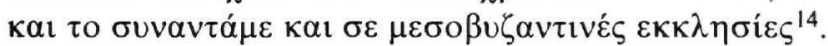

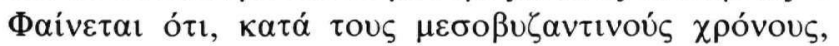

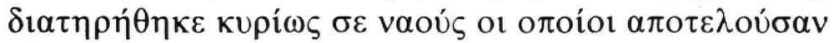

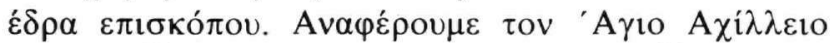
$\Pi \rho \varepsilon ́ \sigma \pi \alpha \varsigma^{15}, \tau \eta \beta \alpha \sigma \iota \lambda \iota \kappa \eta ́ ~ B u \zeta \alpha \rho i ́ o v ~ K \rho \eta ́ \tau \eta \varsigma^{16}, \tau \eta v E \pi 1-$

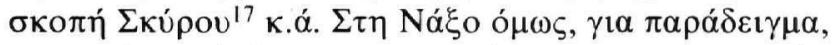

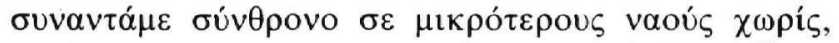

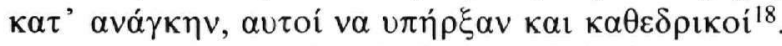

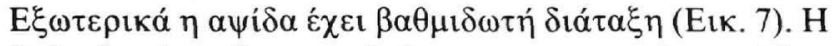

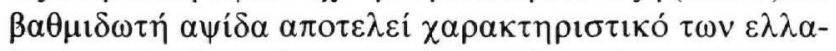

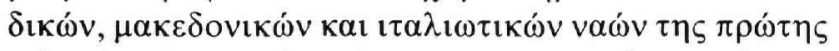

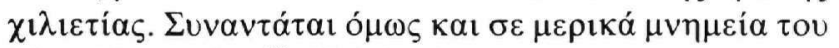

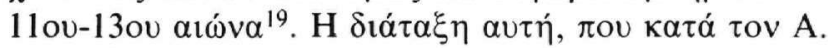

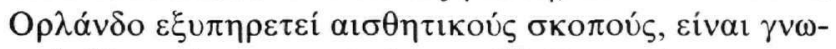

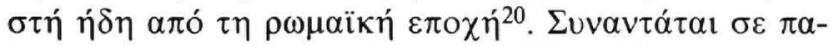

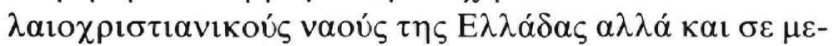

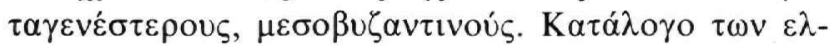

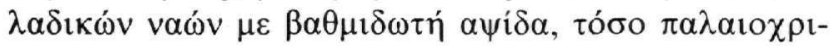

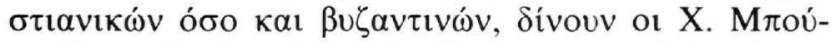

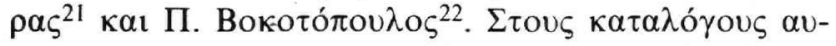

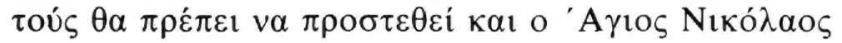
Zaкúvөou.

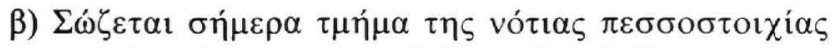

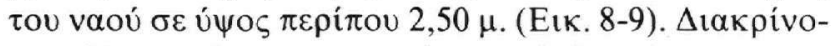

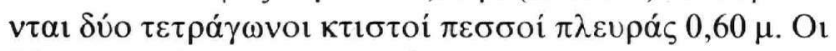

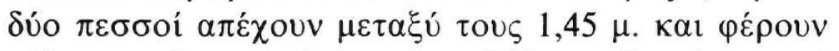

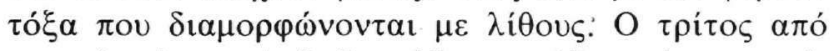

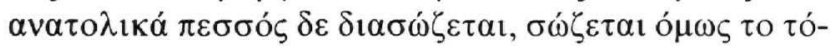

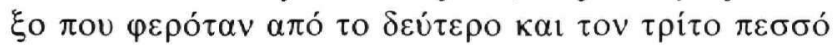

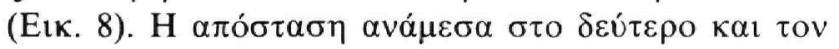

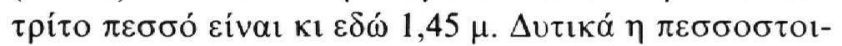

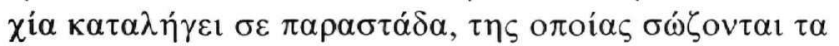

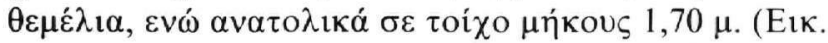

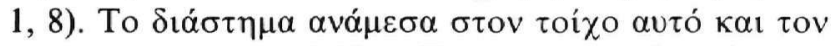

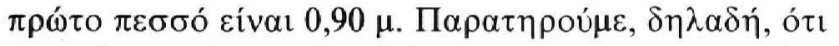

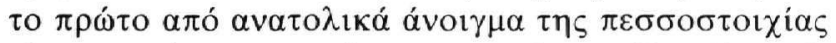

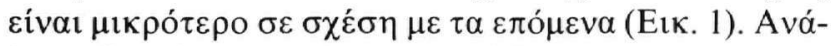

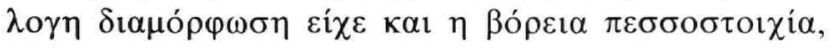

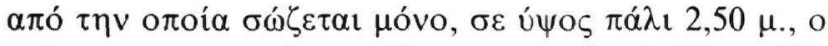

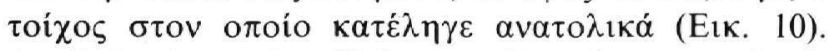

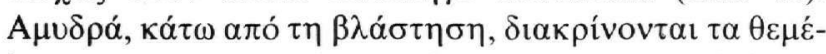

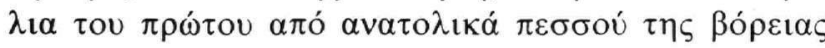
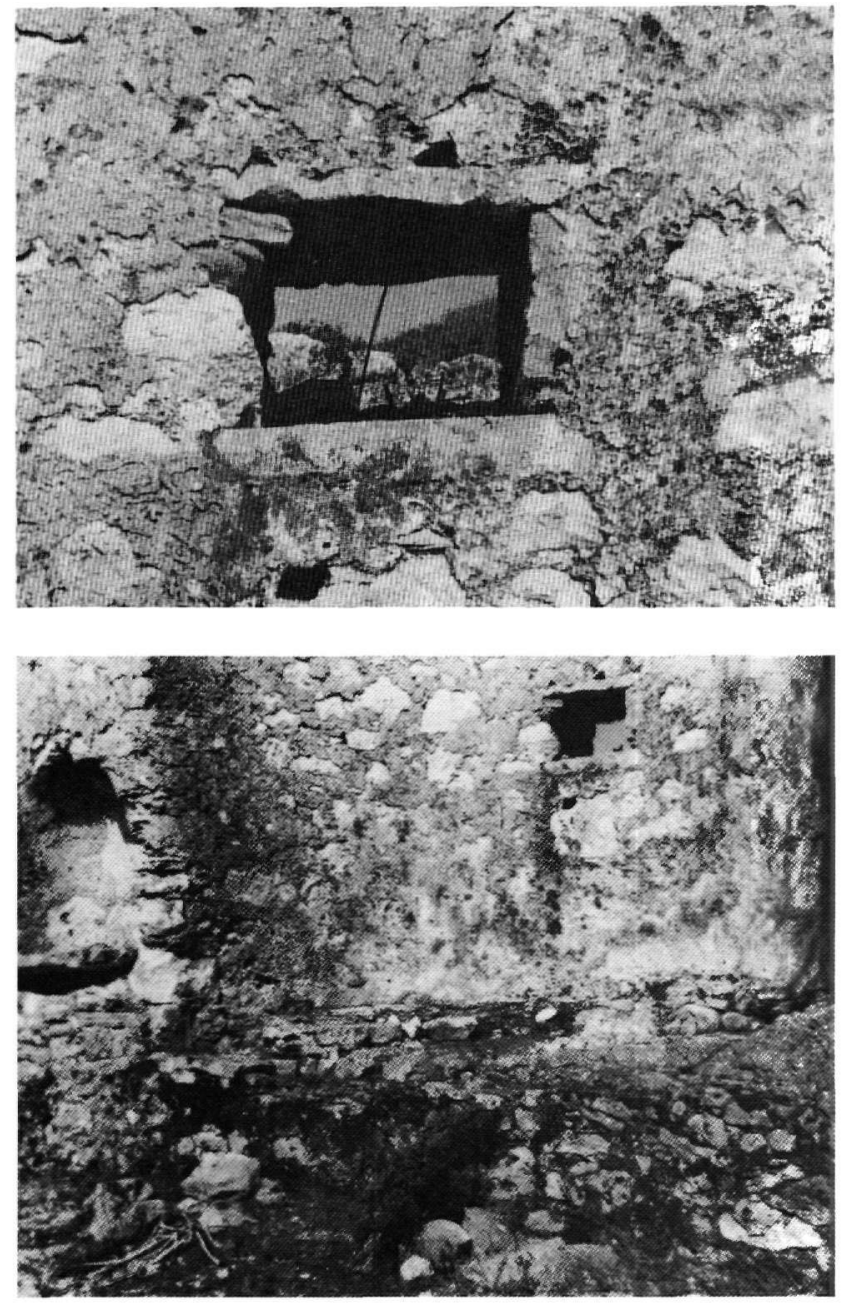

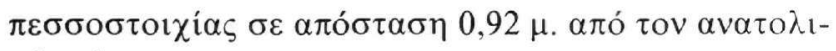

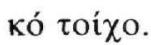

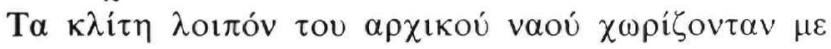

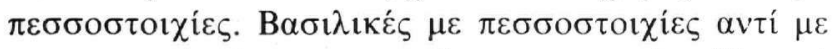

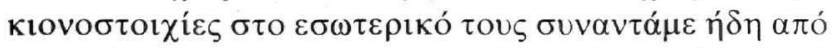

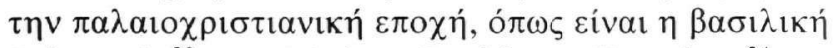

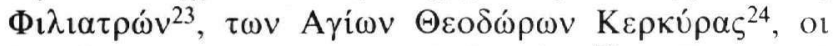

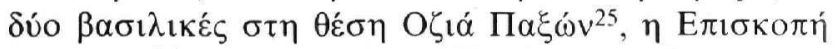

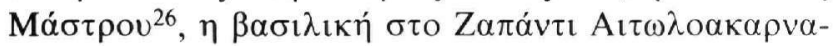

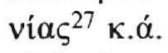

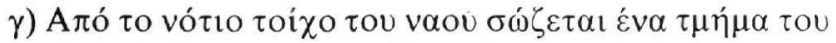

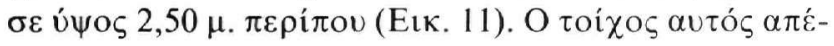

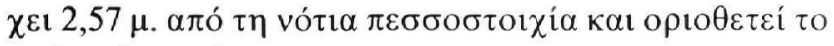
vaó $\alpha \pi$ tó $\tau \alpha$ vó $\tau$.

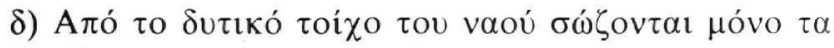

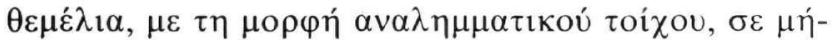

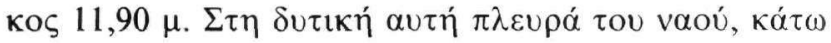

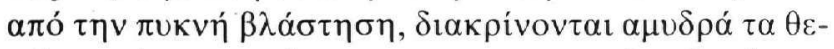

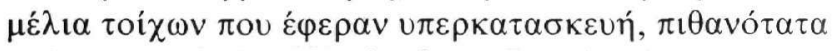

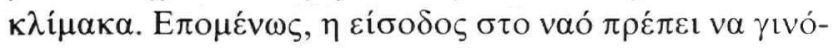



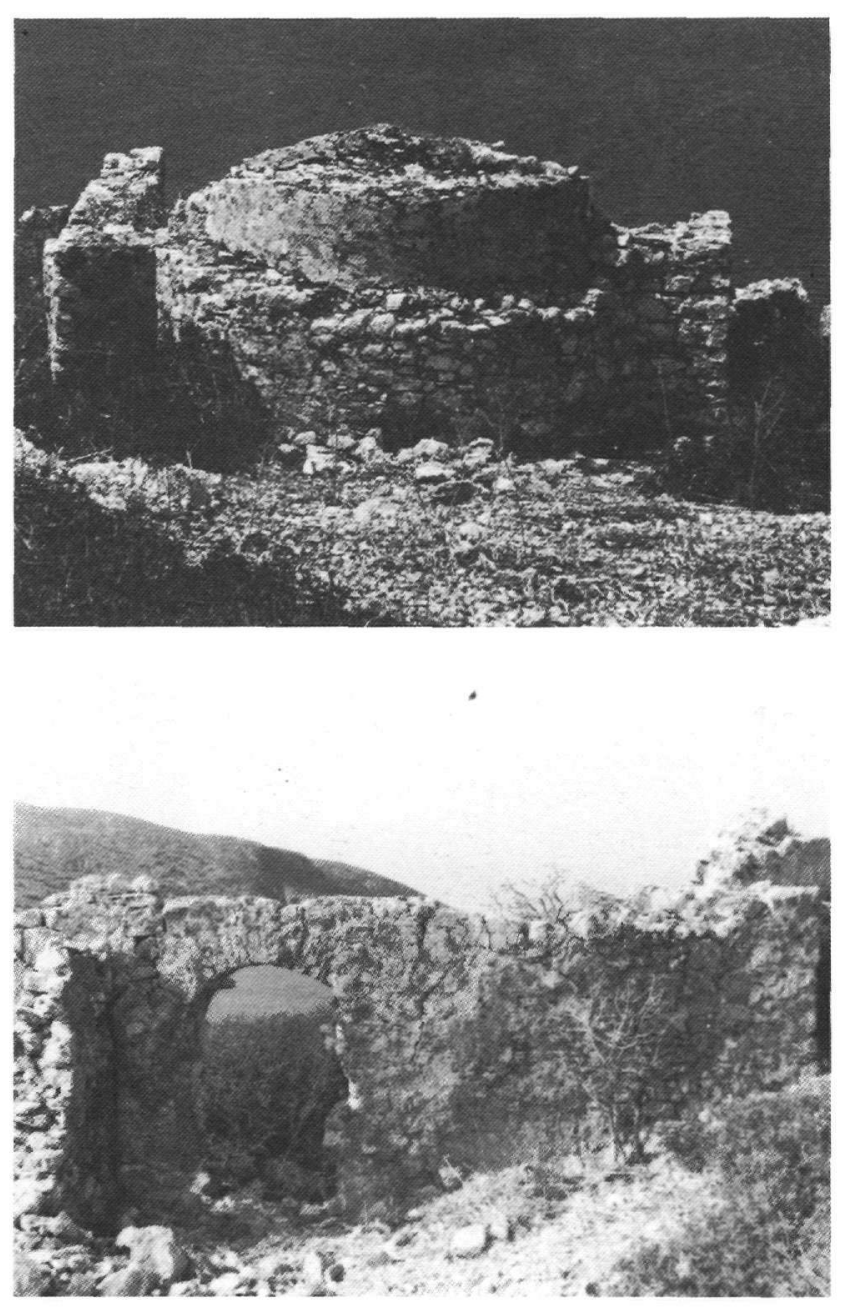

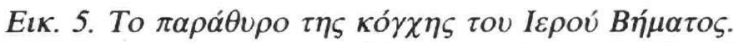

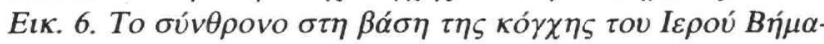

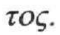

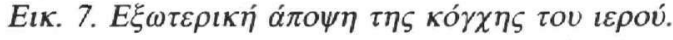

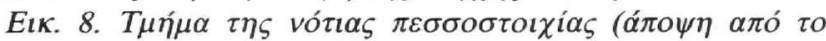

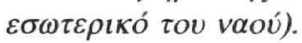

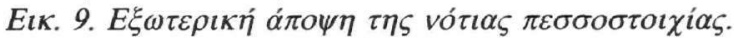

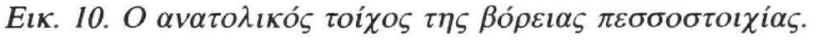

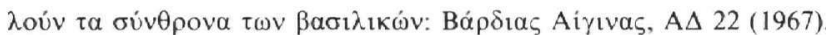

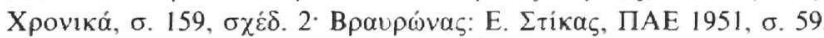

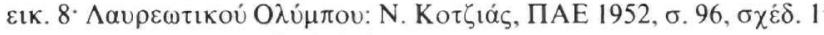

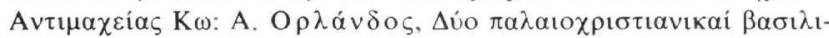

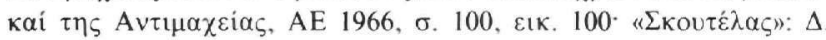

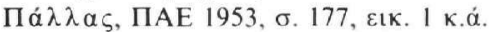

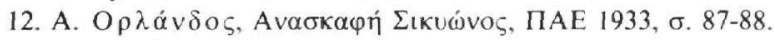

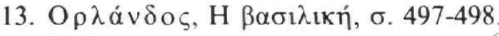

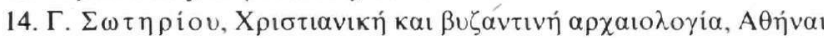
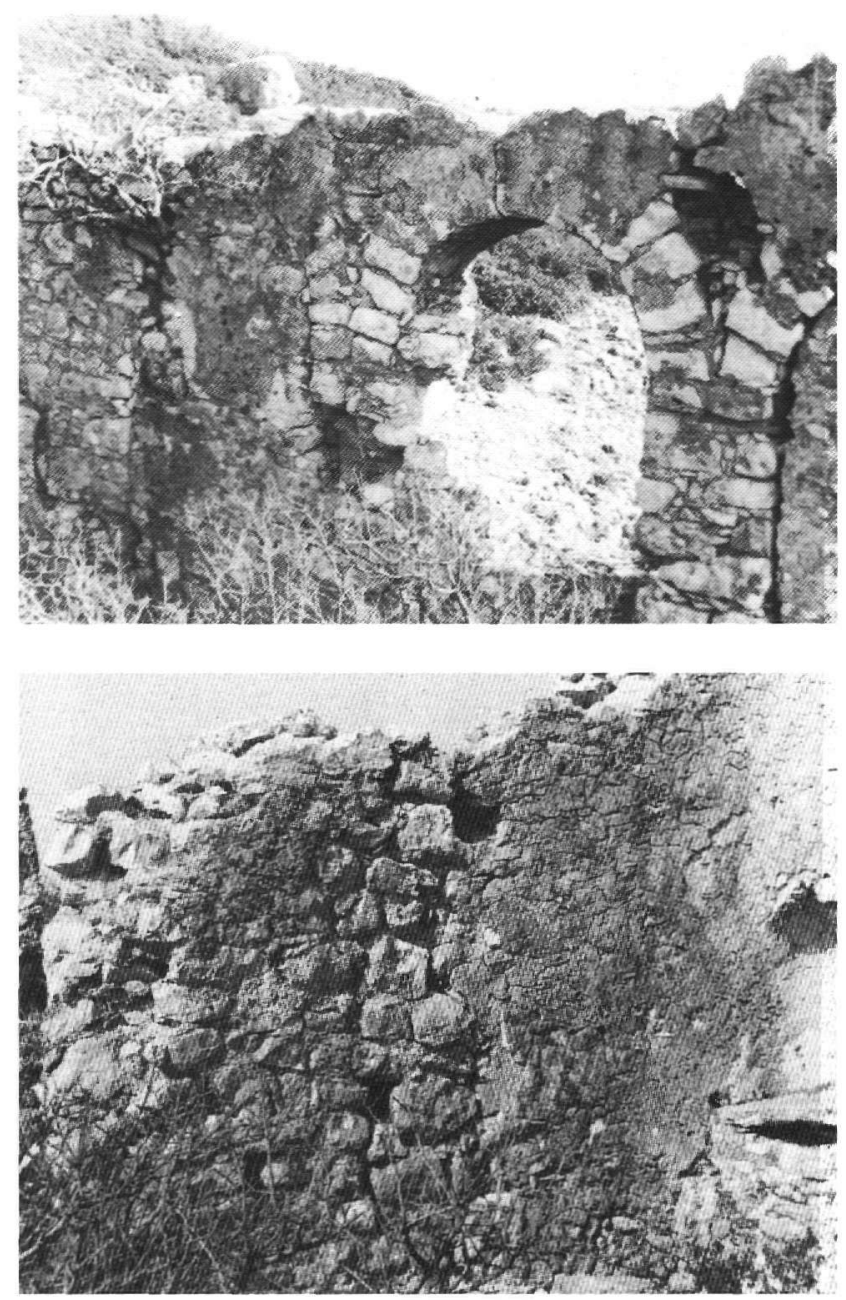

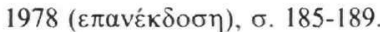

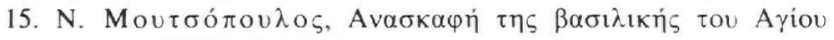

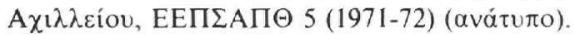

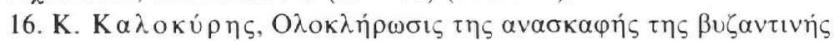

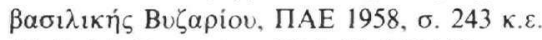

17. R. M. Dawkins, BSA $11(1904 / 5)$

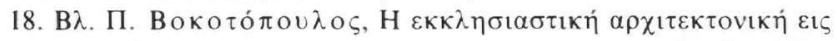

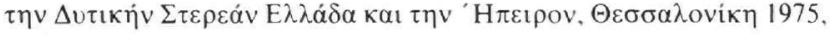

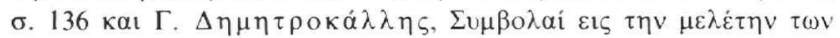

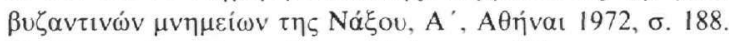

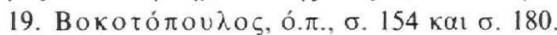

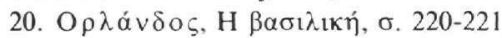

21. B $\lambda$. Ch. Bouras, Zourtsa, une basilique byzantine au Peloponnèse, CahArch XXI (1971), б. 146-147.

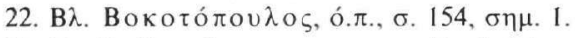

23. D. Pallas, Les monuments paléochrétiens de Grèce decouverts de 1959 à 1973, Roma 1977, б. 188-189.

24. I. П $\alpha \pi \alpha \delta \eta \mu \eta \tau \rho i o v, \mathrm{~K} \varepsilon \rho \kappa X \rho o v 2$ (1952), б. 58. П. K $\alpha \lambda \lambda \iota \gamma \alpha \dot{\alpha}$, ,

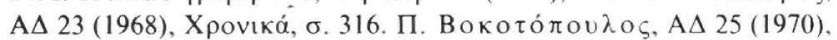

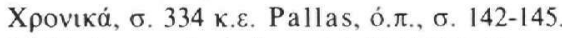

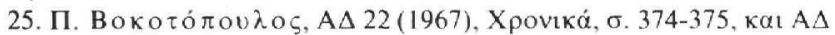

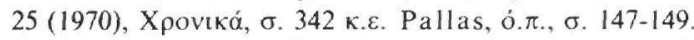

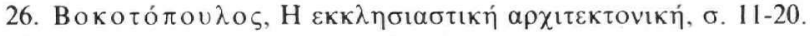

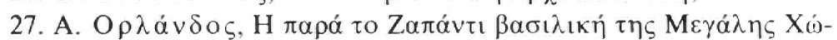




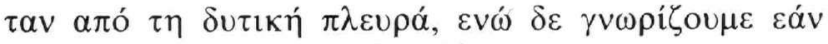

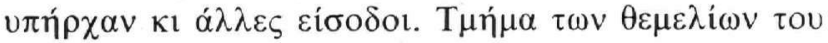

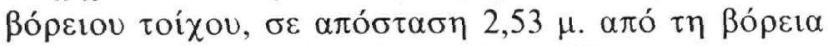

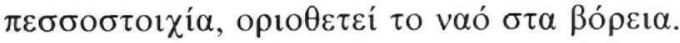

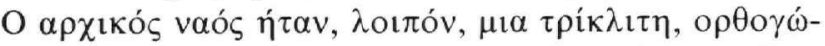

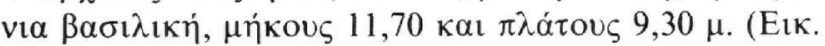

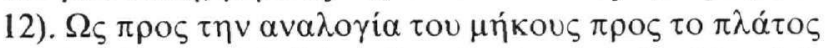

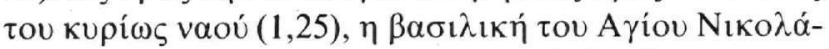

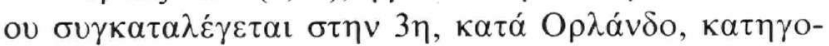

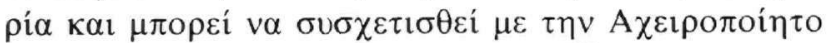

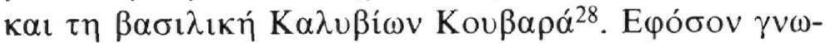

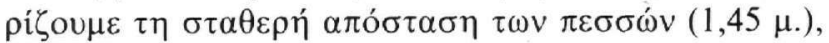

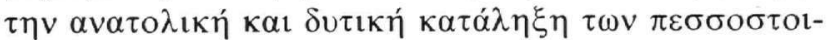

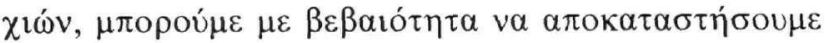

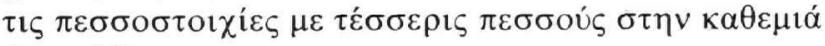
(Еıк. 12).

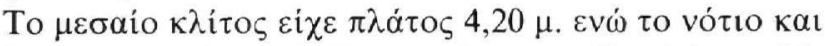

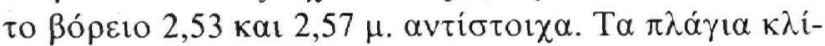

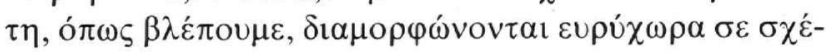

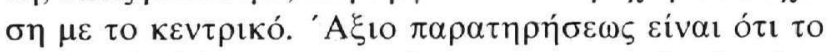

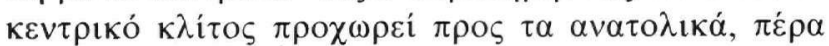

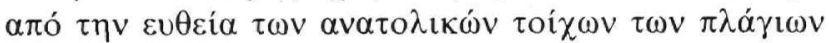

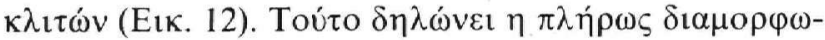

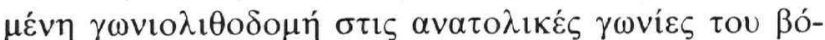


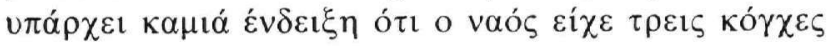

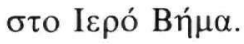

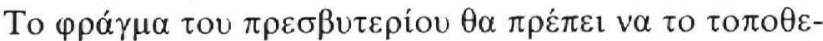

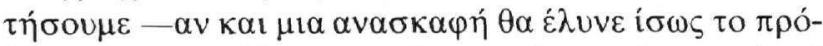

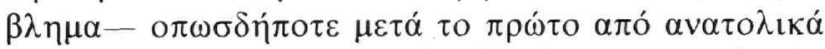

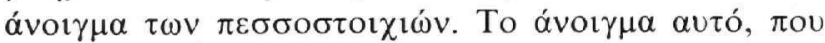

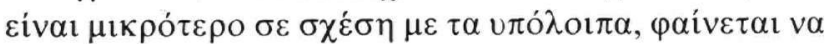

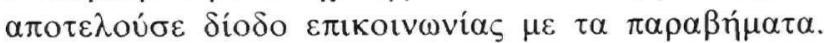

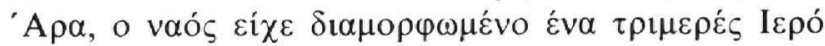
Bí $\mu \alpha$.

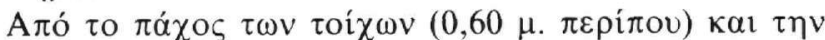

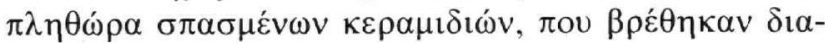

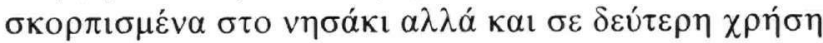

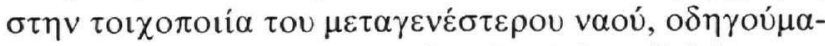

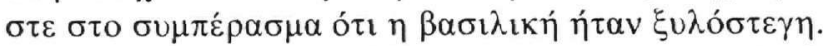

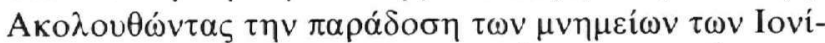

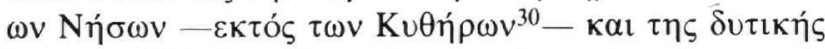

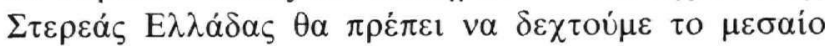

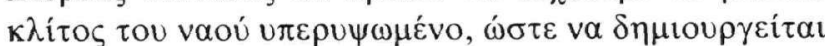

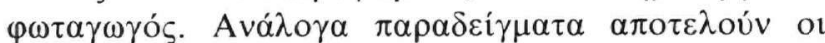
'A

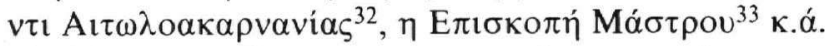

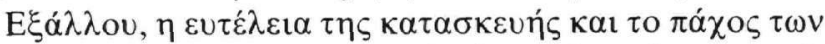

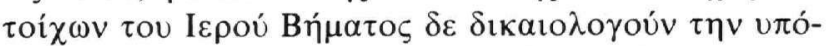

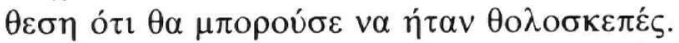

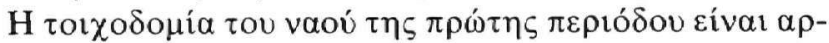

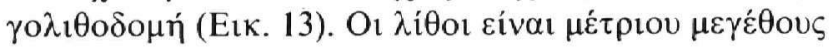
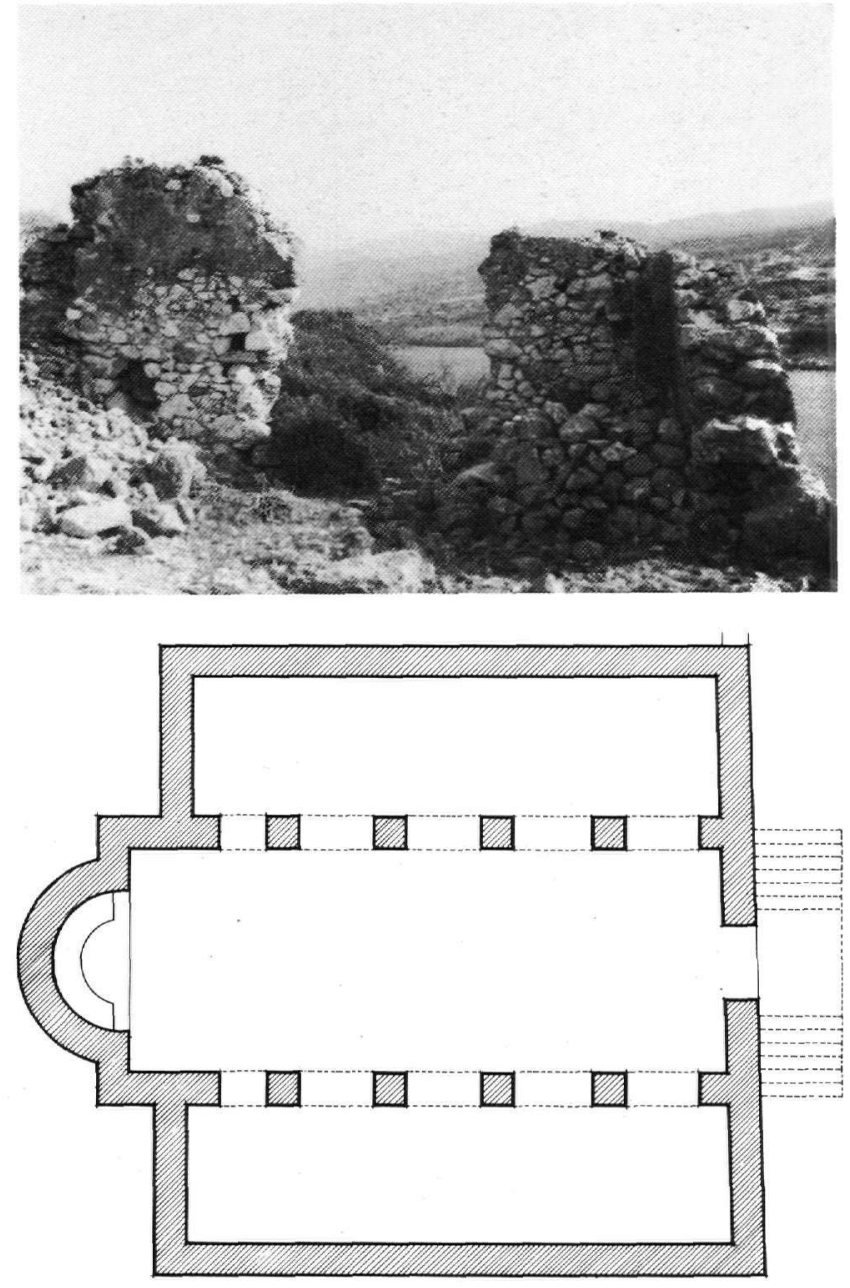

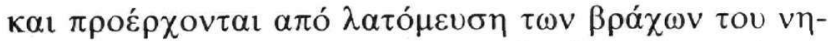

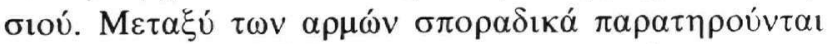

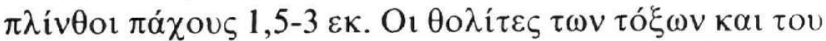

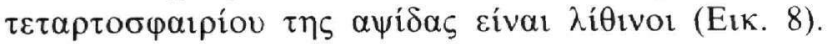

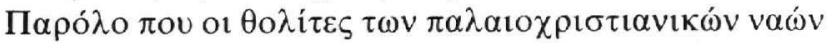

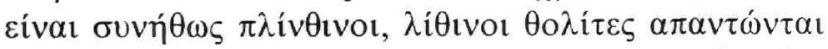

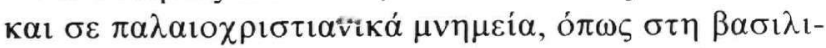

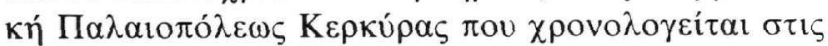

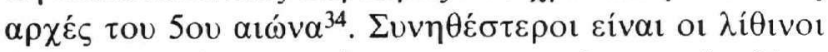

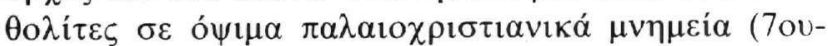

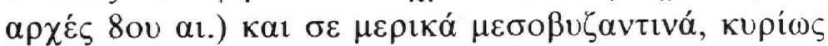

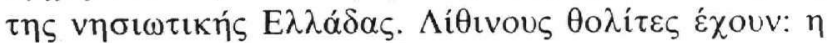

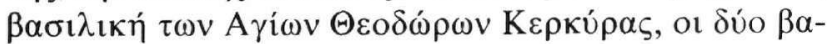

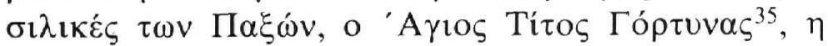

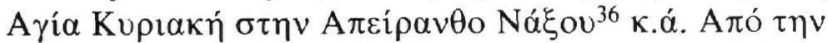

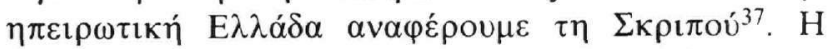

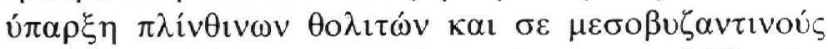

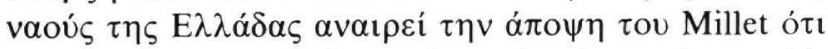

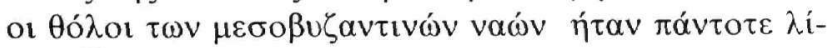
$\theta i v \mathrm{vi}^{38}$. 

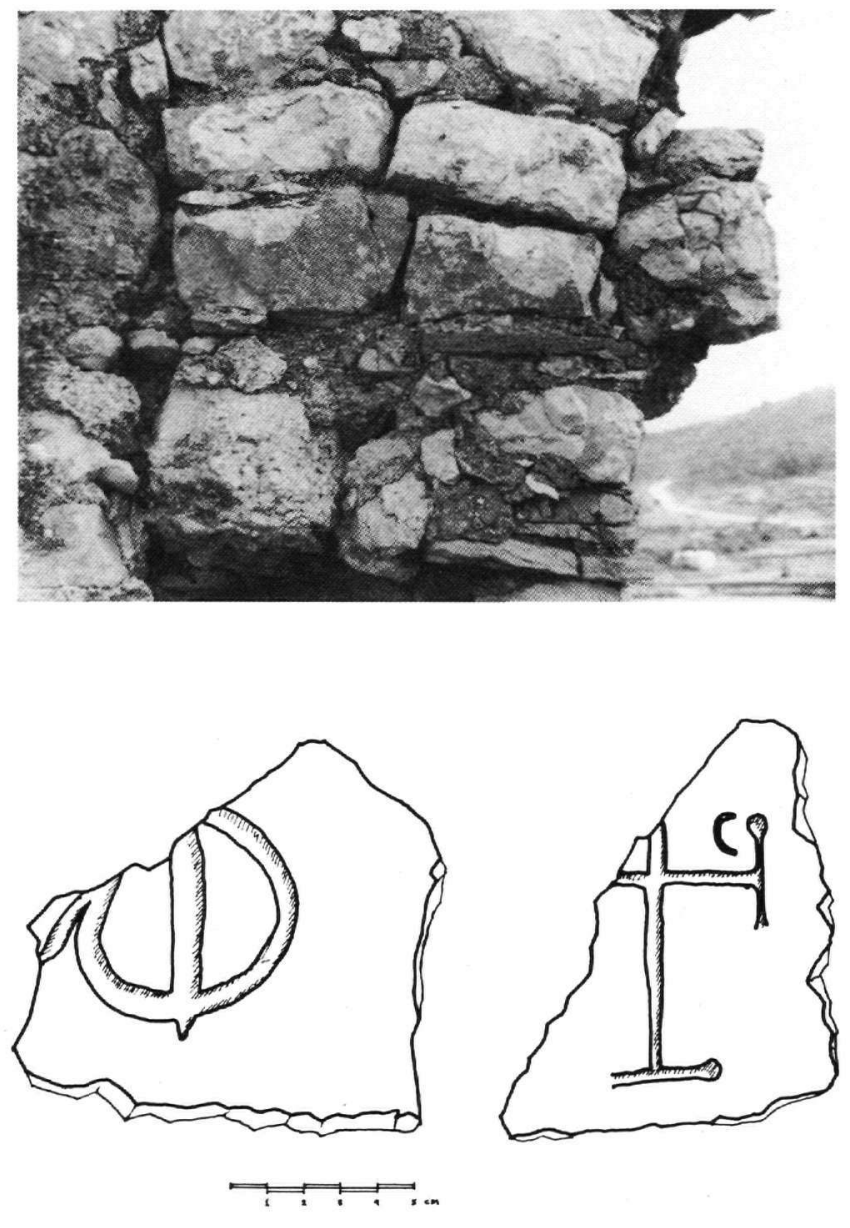

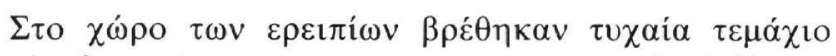

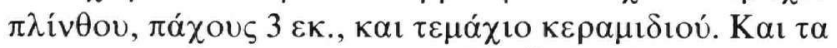

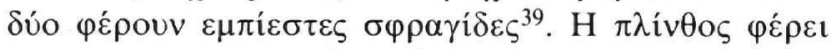

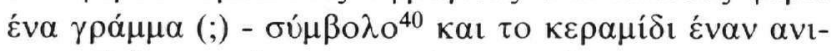

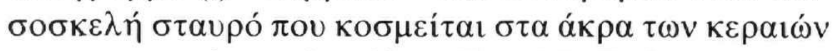

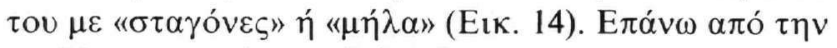

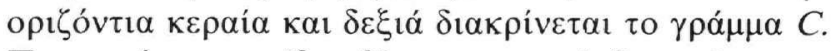

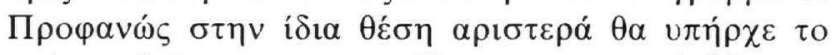

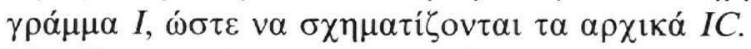

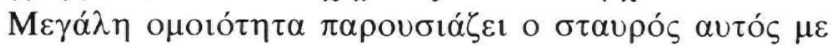

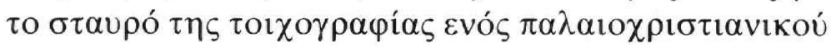

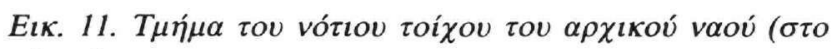
$\mu \varepsilon ́ \sigma o v)$.

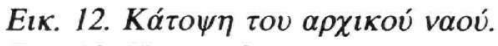

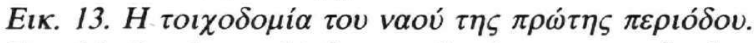

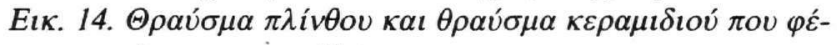

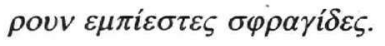

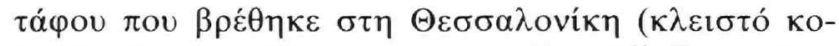

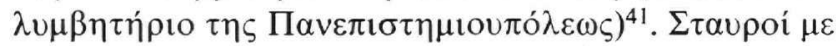

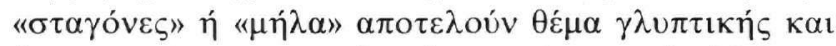

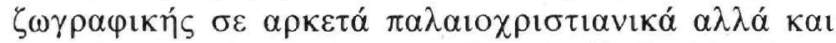

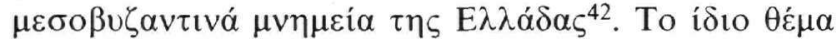

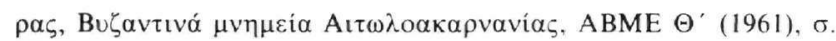
43-53.

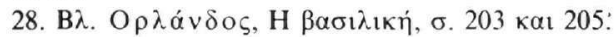

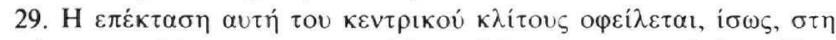

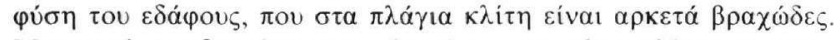

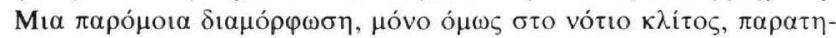

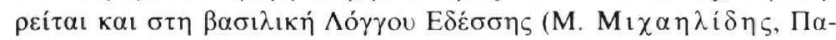

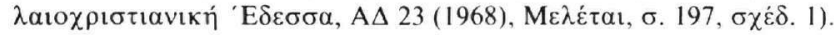

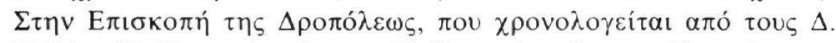

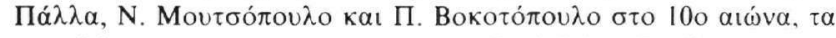

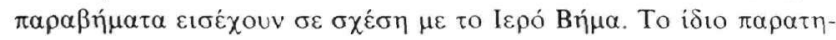

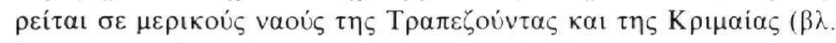

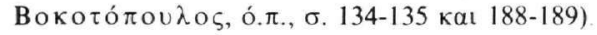

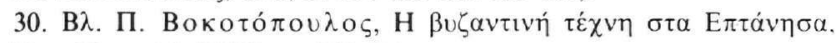

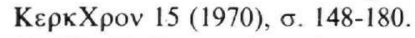

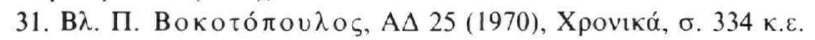

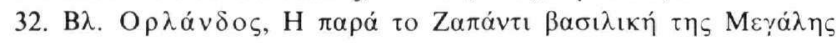

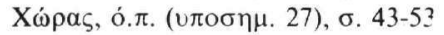

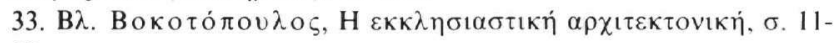
20.

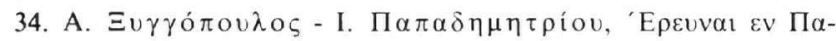

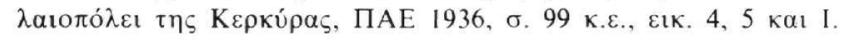

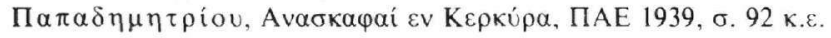

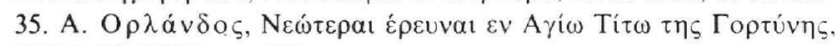
$\operatorname{EEB} \Sigma \Gamma^{\prime}(1926), \sigma .301$.

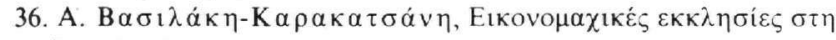
Náło, $\Delta$ XAE, $\pi \varepsilon \rho . \Delta^{\prime} \tau . \Gamma^{\prime}(1962-63), \sigma .52$.

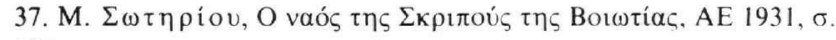
124.

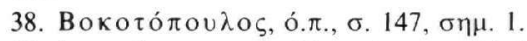

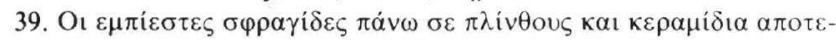

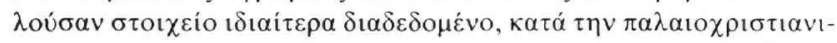

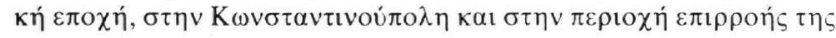

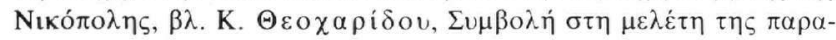

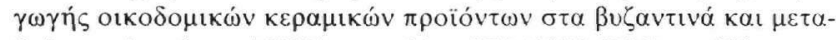

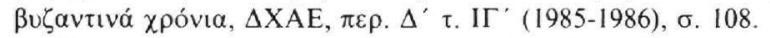

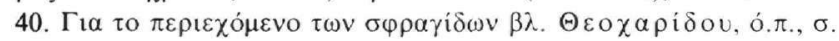
110.

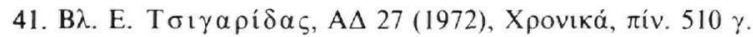

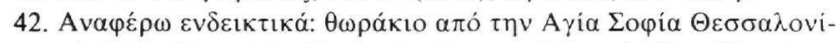
кฤ (M. Kalliga, Die Hagia Sophia von Thessalonike, Würzburg

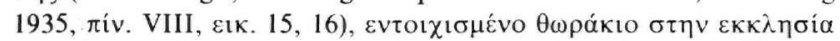

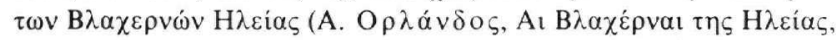

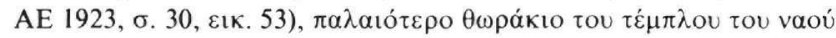

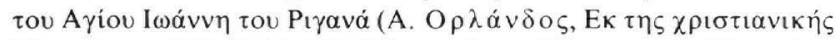

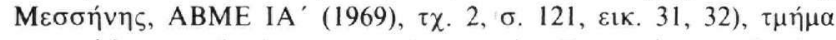

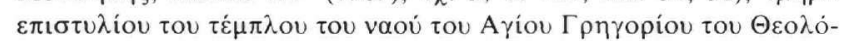

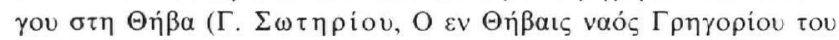

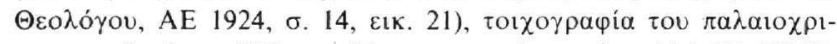

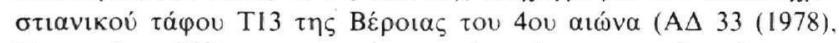

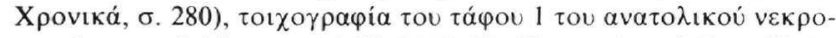

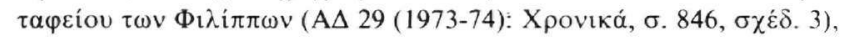




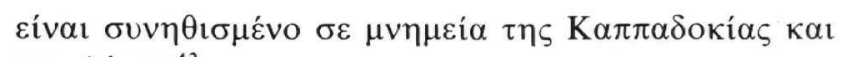
$\tau \eta \varsigma \Delta \dot{\sigma} \sigma \eta \varsigma^{43}$.

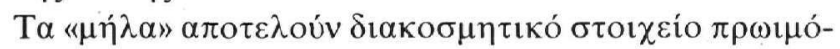

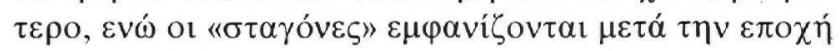

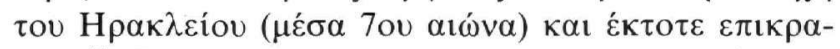

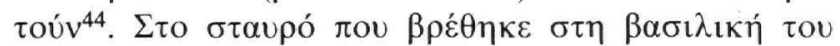

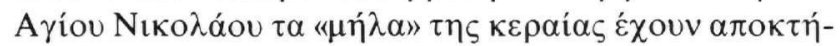

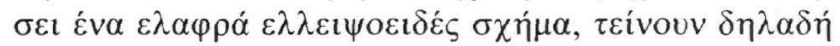

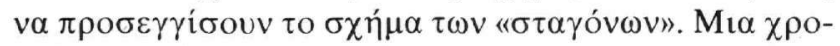

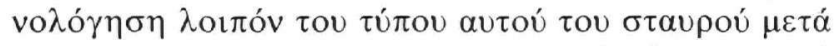

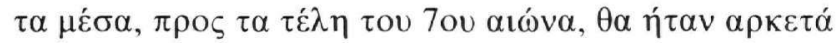
$\pi \imath \theta \alpha \eta \dot{r}$.

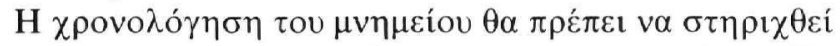

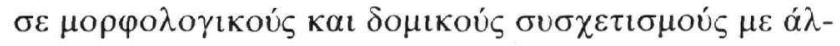

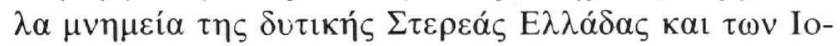

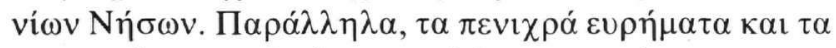

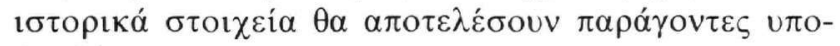

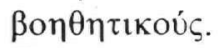

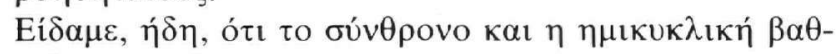

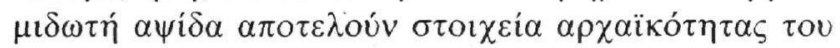

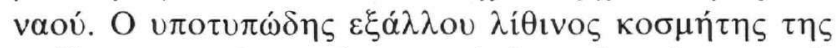

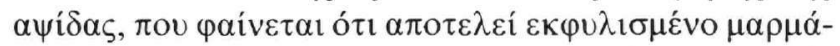

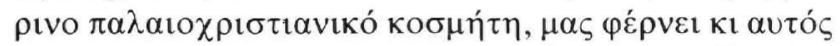

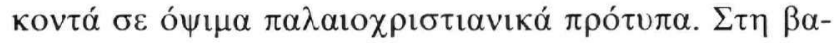

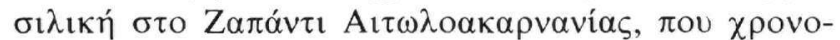

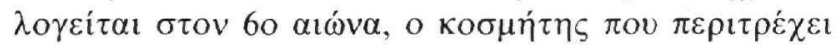

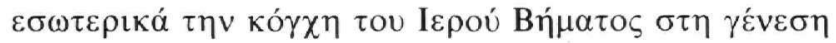

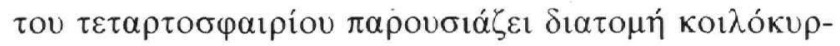

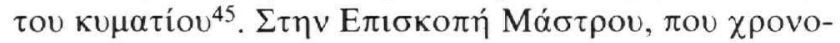

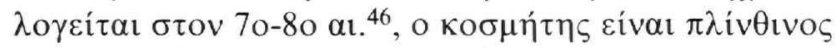

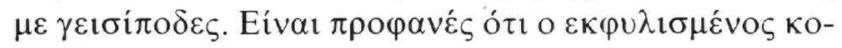

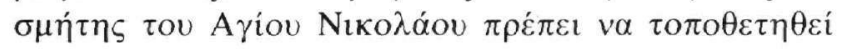

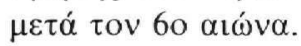

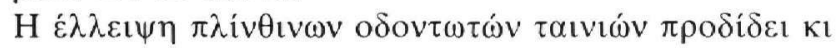

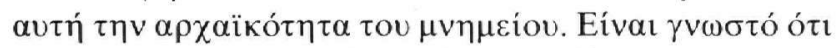

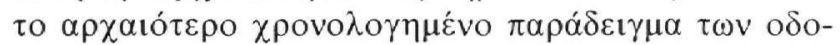

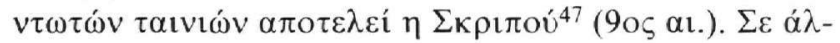

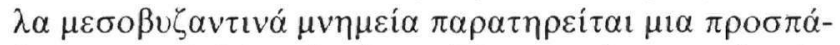

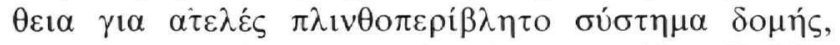

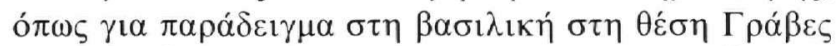

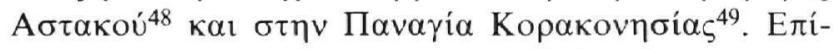

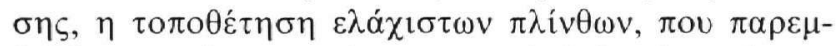

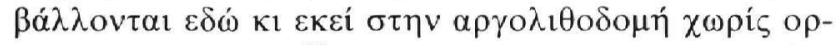

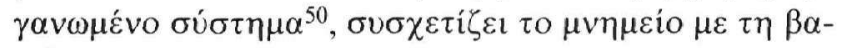

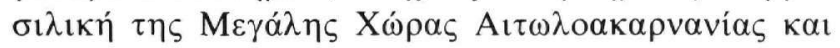

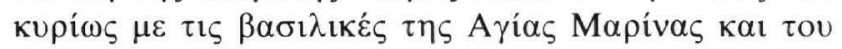

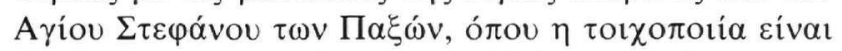

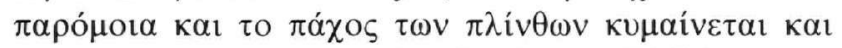

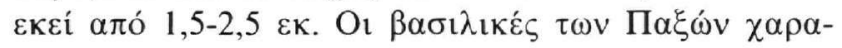

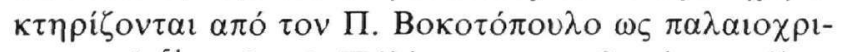

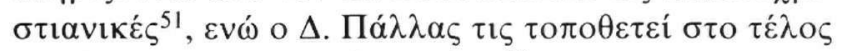

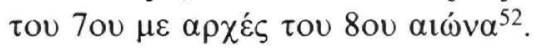

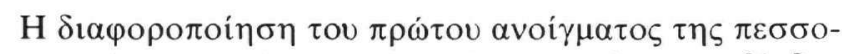

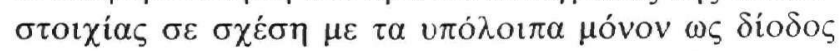

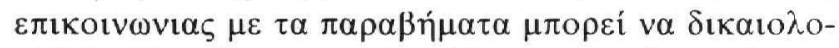

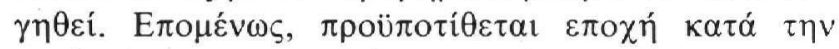

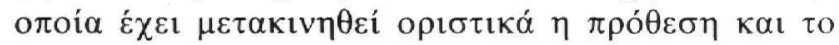

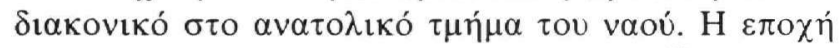

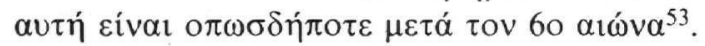

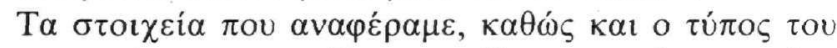

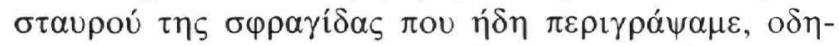

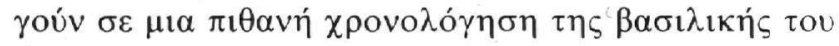

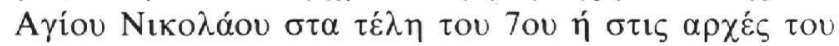

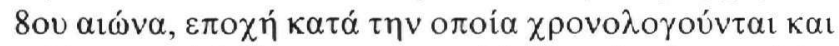

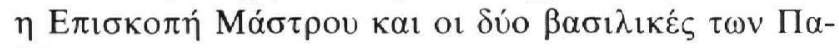
$\xi \dot{\omega} v$.

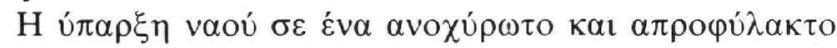

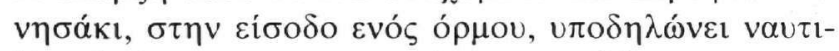

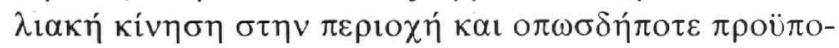

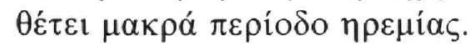

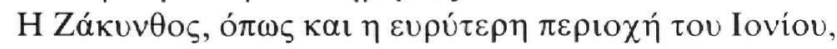

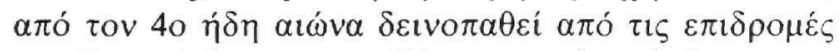

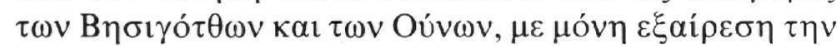

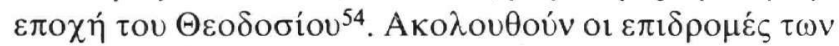

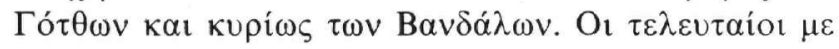

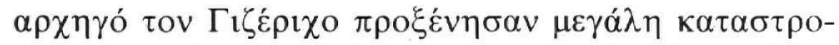

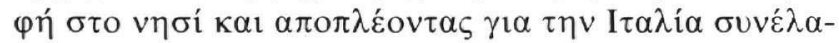

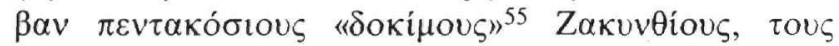

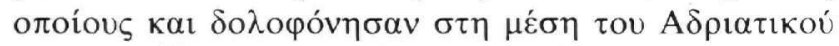

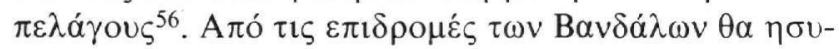

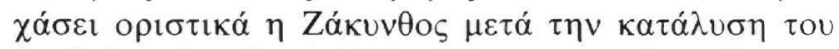

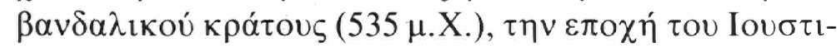

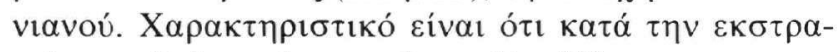

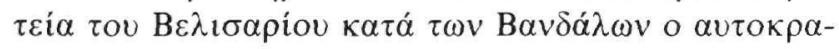

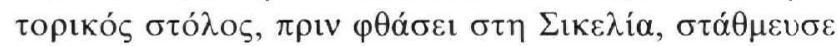

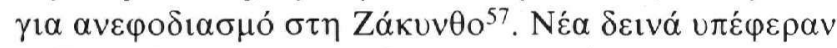

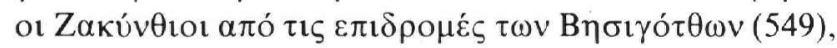

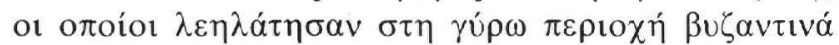

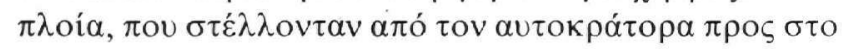

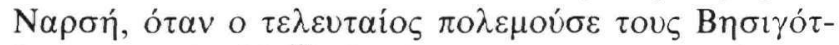

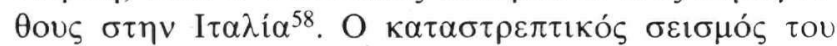

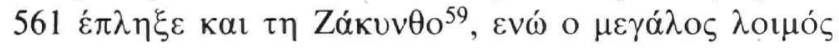

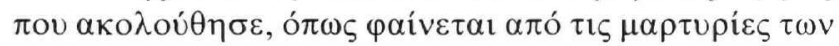

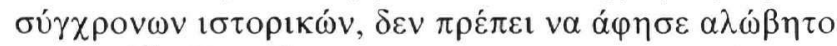

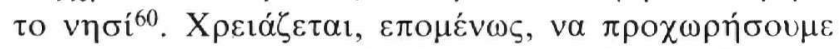

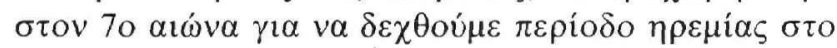

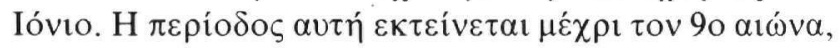

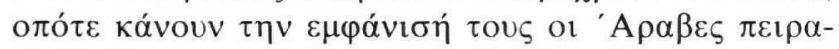

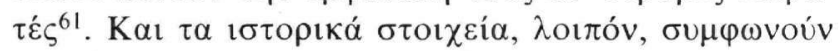

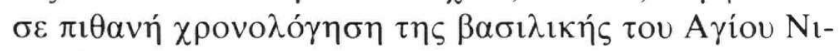

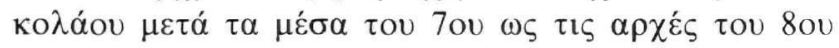
$\alpha \iota \omega ́ v \alpha$.

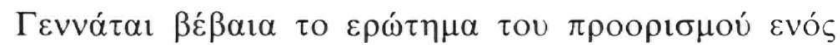

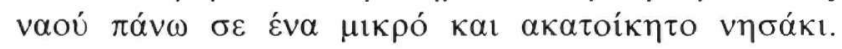




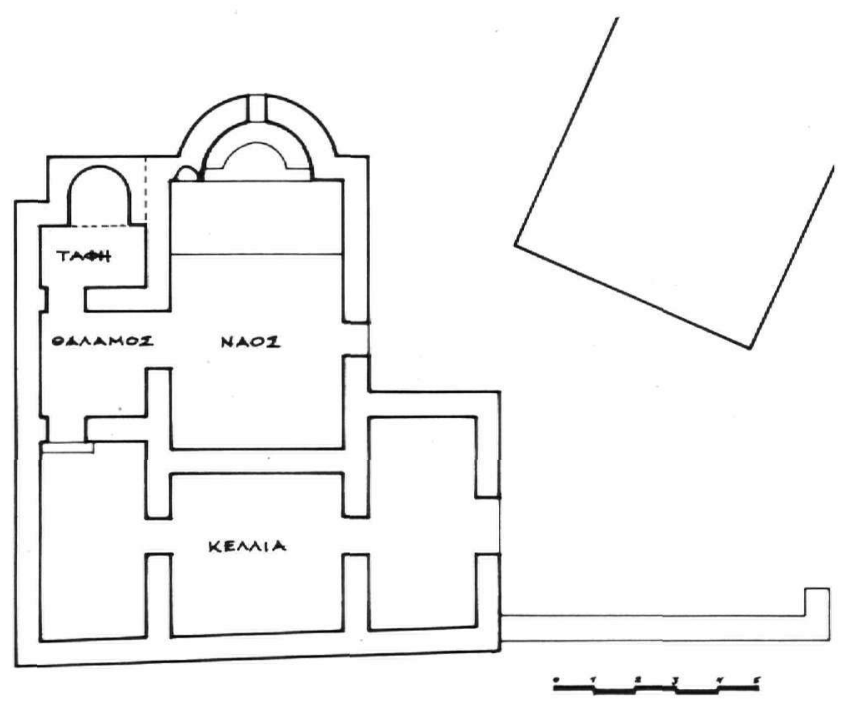

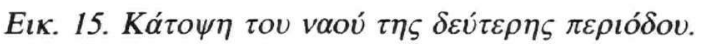

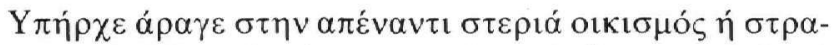

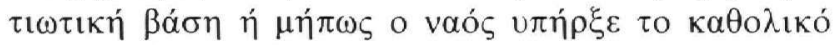

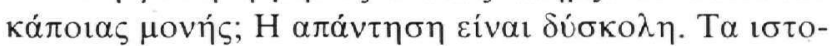

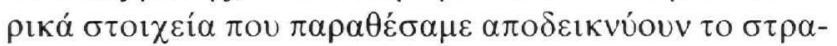

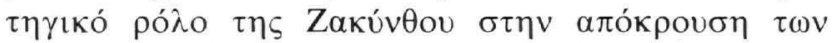

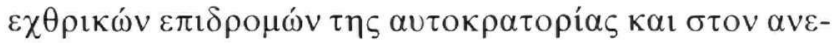

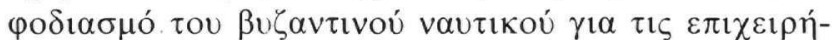

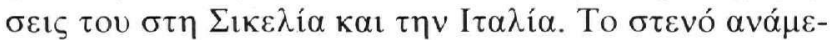

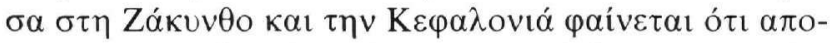

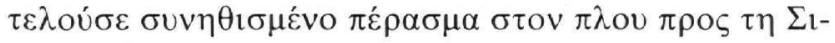

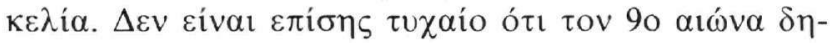

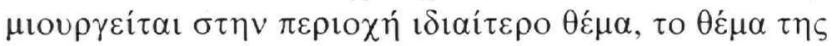

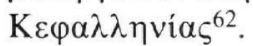

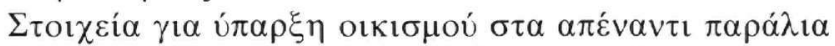

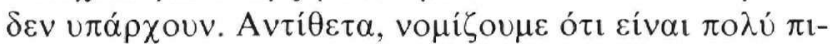

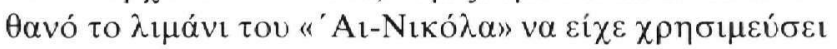

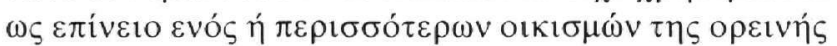

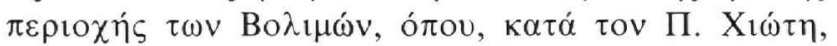

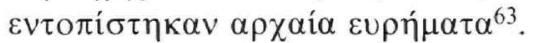

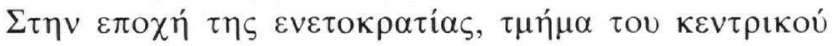

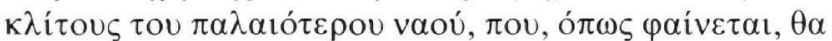

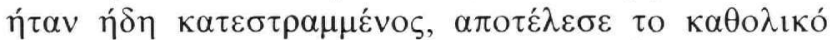

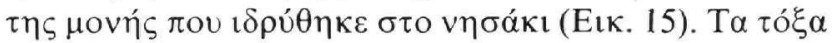

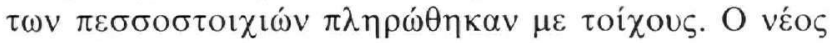

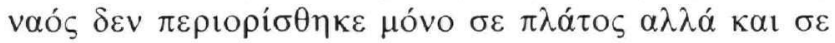

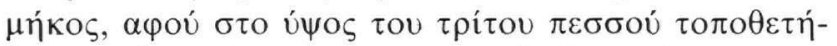

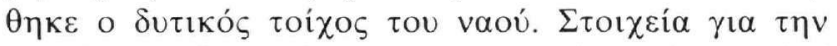

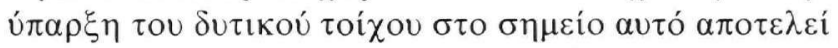

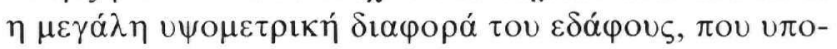

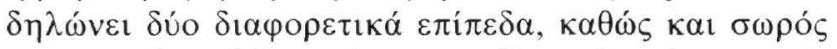

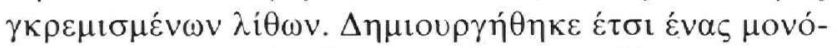

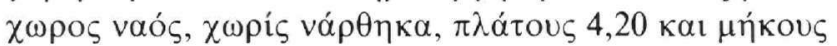
$6,40 \mu$.

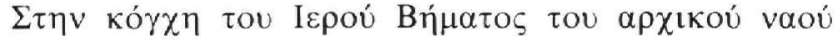

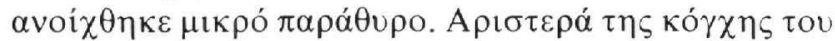

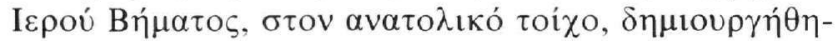

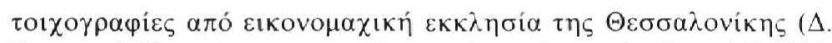

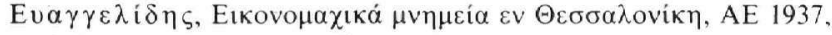

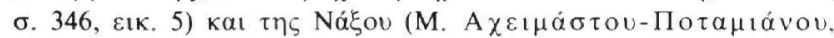

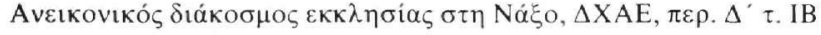
(1984), б. 335, عıк. 7).

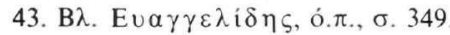

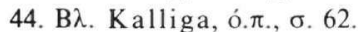

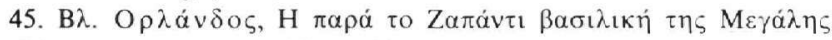

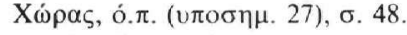

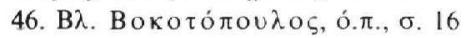

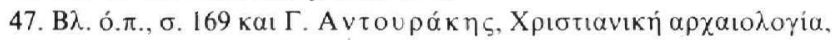

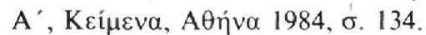

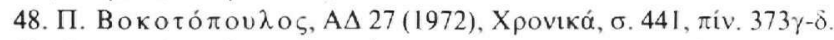

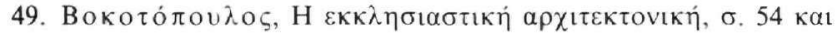
142 .

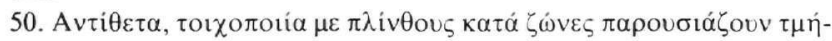

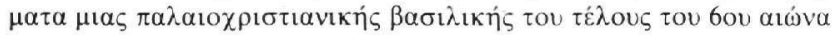

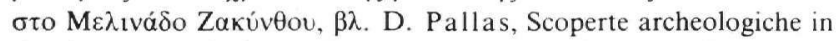
Grecia negli anni 1956-1958, ArchCr, 1959, б. 203, हıк. 17.

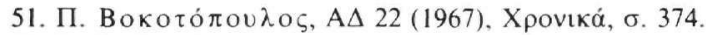

52. D. Pallas, Les monuments paléochrétiens de Grèce decouverts de 1959 à $1973, \sigma .149$.

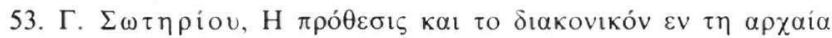

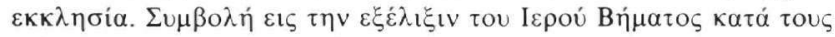

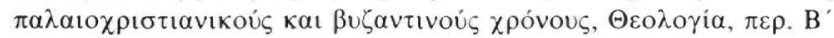

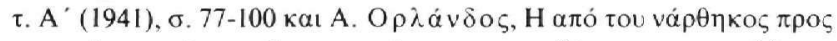

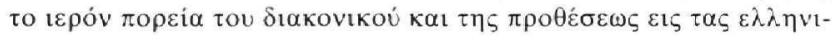
$\sigma \tau \iota \kappa \alpha \dot{\zeta} \beta \alpha \sigma \iota \lambda \iota \kappa \alpha \dot{\alpha}, \Delta \mathrm{XAE}, \pi \varepsilon \rho . \Delta^{\prime} \tau . \Delta^{\prime}(1964-65)$

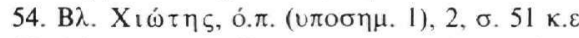

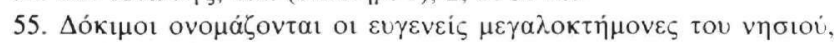
$\beta \lambda . X_{1} \omega \tau \eta \zeta$, ó. $\pi ., \sigma .48$.

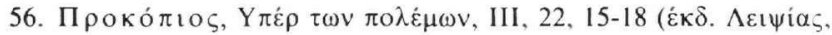

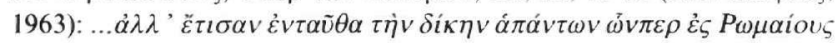

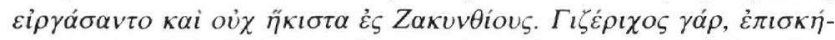

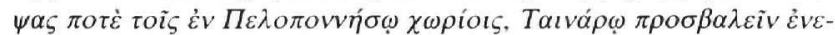

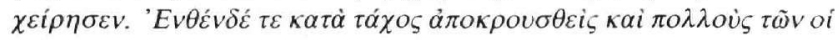

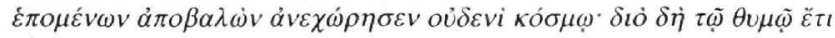

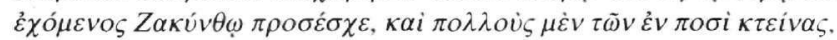

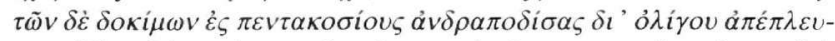

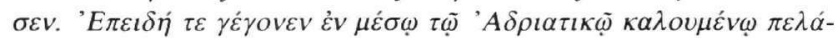
$\gamma \varepsilon l, \dot{\varepsilon} v \tau \alpha \tilde{v} \theta \alpha \kappa \rho \varepsilon o v \rho \gamma \tilde{\eta} \sigma \alpha \varsigma \tau \tilde{\omega} v \pi \varepsilon v \tau \alpha \kappa o \sigma i \omega v \tau \dot{\alpha} \sigma \dot{\omega} \mu \alpha \tau \alpha \pi \alpha v \tau \alpha \chi \tilde{\eta} \tau \tilde{\eta} \zeta$

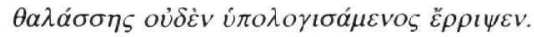

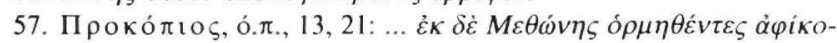

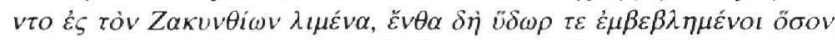

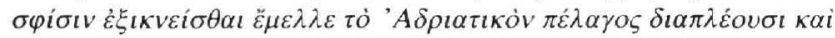
$\tau \dot{\alpha} \ddot{\alpha} \lambda \lambda \alpha \pi \alpha \rho \alpha \sigma \kappa \varepsilon v \alpha \sigma \alpha ́ \mu \varepsilon v o t ~ \check{~} \pi \lambda \varepsilon o v$.

58. 'O.

59. 'O. $\pi .$, VIII, 25,16 к.ع.

60. 'O. $\pi .$, II, 22.

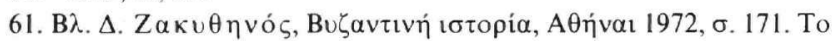

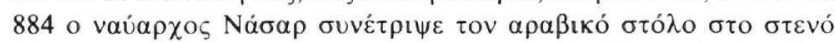

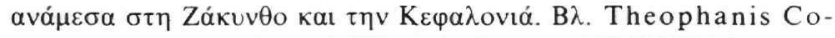

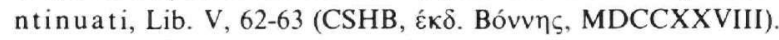

62. B $\lambda$. D. Zakythinos, Le thème de Cephalonie et la defense de l'Occident, L'Hellénisme Contemporain, Athènes 1954, $\sigma$. 303-312.

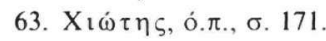




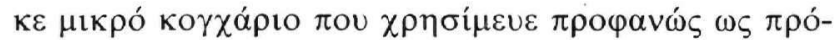

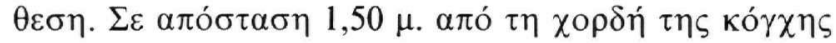

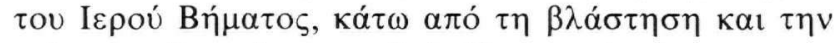

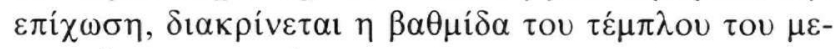
$\tau \alpha \gamma \varepsilon v \varepsilon \dot{\sigma} \sigma \tau \rho \circ \mathrm{v}$ vaoú.

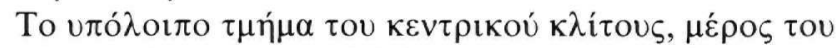

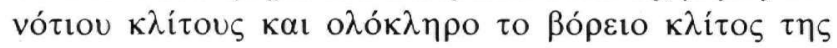

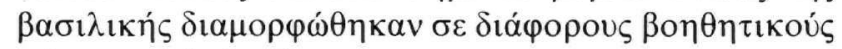

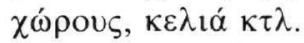

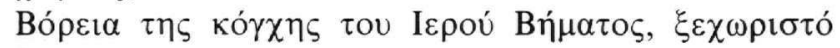

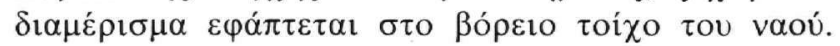

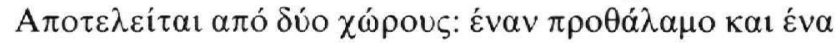

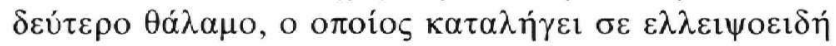

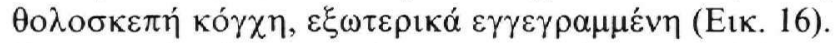

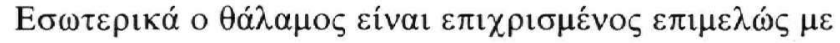

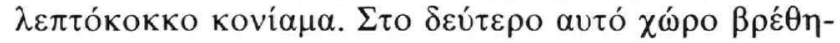

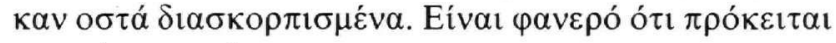

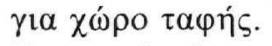

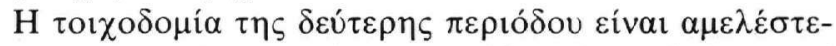

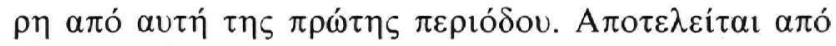

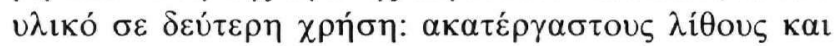

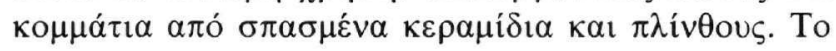

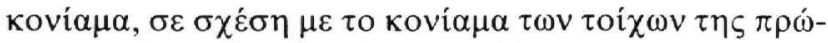

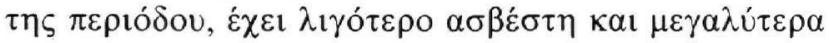
$\kappa \alpha \iota \pi \varepsilon \rho \iota \sigma \sigma o ́ \tau \varepsilon \rho \alpha \beta o ́ \tau \sigma \alpha \lambda \alpha$.

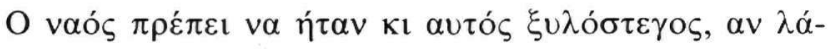

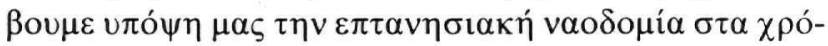

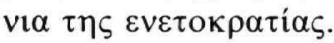

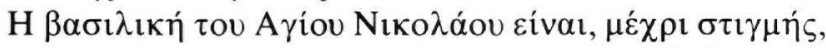

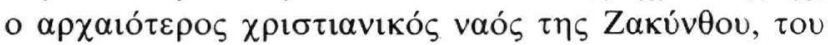

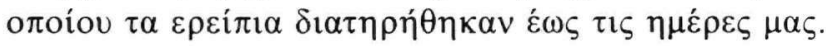

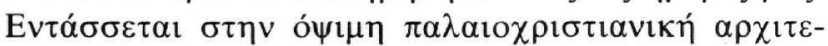

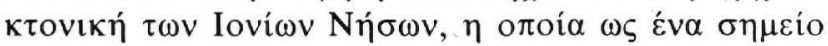

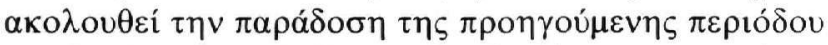

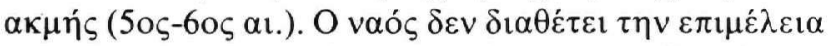

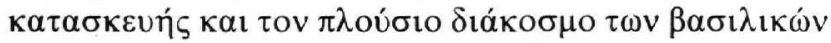

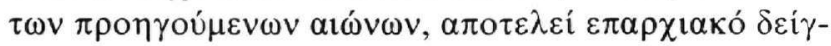

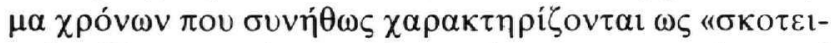

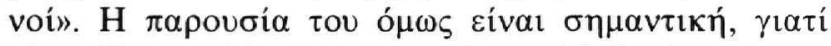

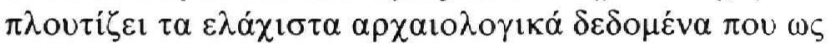

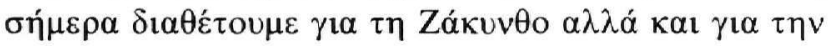

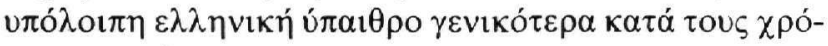
vous avtoús.

\section{Ioanna Stoufi-Poulimenou}

\section{ST. NICHOLAS ON THE ISLAND, ON ZAKYNTHOS. AN UNKNOWN EARLY CHRISTIAN BASILICA}

$T_{1}$ uninhabited island at the northeast end of the island of Zakynthos. During the last period of its existence, from the 15 th century to 1843 , the monument was used as the church of a monastery. The surrounding area of this monastery is now in ruins.

Two phases can be distinguished in the construction of the monument. Unworked stones and a few bricks were used for the bema vault, the arcades, and part of the south wall; and unworked stones, broken tiles and bricks were employed in second use for the masonry between the arcades and the other walls of the monument.

The earlier church was a three-aisled rectangular basilica $(11.70 \times 9.30 \mathrm{~m}$. $)$ without a narthex. The aisles were separated by piers, from which the arcades were sprung. Since the walls are only $60 \mathrm{~cm}$. thick, we may tentatively conclude that the church of St. Nicholas was a timberroofed basilica. The monument has a number of features of the Early Christian period: a) the synthronon in the apse (the synthronon is of composite type according to A. Orlandos); b) the step of the semi-circular apse; c) the use of unworked stones and a few bricks for the masonry; and d) the absence of any kind of ceramic ornament. The almost unformed cornice of the apse is reminiscent of Early Christian churches.

A monastery was built on the ruins of this monument during the Venetian occupation of Zakynthos. Its church was part of the central aisle of the basilica. The island enjoyed a period of peace after the second half of the 6th century. In view of this, and of the morphological features of the monument, the basilica of St. Nicholas may be dated to the end of the 7th or the beginning of the 8th centuries. Two basilicas on the island of $\mathrm{Pa}-$ xoi, which date from this same period, have close morphological similarities with the monument under discussion.

It might be suggested, therefore, that the basilica of St. Nicholas is the only remaining Early Christian church on the island of Zakynthos. 